NBER WORKING PAPER SERIES

\title{
NONPARAMETRIC PRICING OF INTEREST \\ RATE DERIVATIVE SECURITIES
}

Yacine Airt-Sahalia

Working Paper 5345

\author{
NATIONAL BUREAU OF ECONOMIC RESEARCH \\ 1050 Massachusetts Avenue \\ Cambridge, MA 02138 \\ November 1995
}

This paper is part of my Ph.D dissertation at the MIT Department of Economics. I am very grateful to my advisors Jerry Hausman, Andrew Lo and Whitney Newey, as well as John Cox, Lars Hansen, Jose Scheinkman and Daniel Stroock for suggestions. The comments of Peter Robinson and three referees led to considerable improvement of the paper. I thank seminar participants at Berkeley, British Columbia, Carnegie-Mellon, Chicago, Columbia, Harvard, LSE, Michigan, MIT, Northwestern, Princeton, Stanford, Toulouse, ULB, Washington University, Wharton and Yale for comments. Financial support from École Polytechnique and MIT Fellowships is also gratefully acknowledged. All errors are mine. This paper is part of NBER's research program in Asset Pricing. Any opinions expressed are those of the author and not those of the National Bureau of Economic Research.

(c) 1995 by Yacine Aït-Sahalia. All rights reserved. Short sections of text, not to exceed two paragraphs, may be quoted without explicit permission provided that full credit, including ( $)$ notice, is given to the source. 


\title{
NONPARAMETRIC PRICING OF INTEREST \\ RATE DERIVATIVE SECURITIES
}

\begin{abstract}
We propose a nonparametric estimation procedure for continuous-time stochastic models. Because prices of derivative securities depend crucially on the form of the instantaneous volatility of the underlying process, we leave the volatility function unrestricted and estimate it nonparametrically. Only discrete data are used but the estimation procedure still does not rely on replacing the continuous-time model by some discrete approximation. Instead the drift and volatility functions are forced to match the densities of the process. We estimate the stochastic differential equation followed by the short term interest rate and compute nonparametric prices for bonds and bond options.
\end{abstract}

Yacine Aït-Sahalia

Graduate School of Business

University of Chicago

1101 East 58th Street

Chicago, IL 60637

and NBER 


\section{Introduction}

Derivative securities are contingent claims whose payoffs depend upon another asset's payoff. Stock options, futures, swaps, caps, floors, bonds, callable bonds, convertibles, bond options, all fall into this category. Derivative securities are widely traded both over the counter and on exchanges, and more often than not the volume traded is much larger for the derivative securities than the underlying assets that sustain them.

The theory of derivative security pricing relies essentially on continuous-time arbitrage arguments since the pioneering Black and Scholes (1973) paper. As further demonstrated by the work of Merton (e.g., Merton $(1973,1990))$ pricing derivatives in the theoretical finance literature is generally much more tractable --as well as elegant-- in a continuous-time framework than through binomial or other discrete approximations. The empirical option pricing literature however has not followed suit. It is typical there to abandon the continuous-time model altogether when estimating derivative pricing models. This paper develops tools to estimate the actual model used in the theoretical construction (which is in continuous-time) by using only the data available (which are in discrete-time). We will then estimate the short term interest rate process and subsequently price bonds and bond options.

The underlying process of interest $\left\{r_{1}, t \geq 0\right\}$ is a diffusion represented by the Itô stochastic differential equation:

$$
\text { (1.1) } \mathrm{dr}_{\mathrm{t}}=\mu\left(\mathrm{r}_{\mathrm{t}}\right) \mathrm{dt}+\sigma\left(\mathrm{r}_{\mathrm{t}}\right) \mathrm{dW}
$$

where $\left\{\mathrm{W}_{\mathrm{t}}, \mathrm{t} \geq 0\right\}$ is a standard Brownian motion. The functions $\mu($.$) and \sigma^{2}($.$) are$ respectively the drift (or instantaneous mean) and the diffusion (or instantaneous variance) functions of the process. It has long been recognized in the finance literature that one of the most important features of (1.1) for derivative security pricing is the specification of the function $\sigma^{2}($.).

As a consequence every model has tried to specify $\sigma^{2}$ (.) correctly. To price interest rate derivatives, Vasicek (1977) specifies that the instantaneous volatility of the spot rate process is constant. The Cox-Ingersoll-Ross (1985b) (CIR thereafter) model of the term structure assumes that the instantaneous variance is a linear function of the level of the spot rate $\mathrm{r}$, so the standard deviation is a square-root. There are many other models in the literature for the instantaneous variance of the short term interest rate, all being in general mutually exclusive (see Table 1). In the absence of any theoretical rationale for adopting 
one particular specification of the diffusion over another, the question must ultimately be decided by taking the models to the data.

The statistical literature contains methods appropriate for a continuous-time record of observations (e.g., Basawa and Prakasa-Rao (1980), Florens-Zmirou (1993)). Nelson (1990) studies the limiting behavior of discrete approximations as the sampling interval goes to zero. Banon (1978) examined the estimation of the drift when the diffusion is either a constant or a known function. With discrete data, an approach based on maximumlikelihood estimation of the parameters is due to Lo (1988). This method however requires that a partial differential equation be solved numerically for each maximum-likelihood iteration --except in the few cases where it is known explicitly: see Pearson and Sun (1994) for an application to the CIR model. Duffie and Singleton (1993) propose a method of moments where sample paths are simulated for given parameter values and the moments computed (see also Gouriéroux, Monfort and Renault (1993)). Parameter estimates make the simulated moments close to the sample moments. This requires that new sample paths be simulated every time the parameters are adjusted. For a particular parametrization of $\mu($. and $\sigma^{2}($.$) , this method could be applied with sample paths simulated from (1.1). Hansen$ and Scheinkman (1995) derive theoretical moment conditions characterizing the infinitesimal generators of Markov processes.

A commonly used method to estimate (1.1) consists in first parametrizing $\mu($.$) and$ $\sigma^{2}($.$) , then discretizing the model in order to estimate the parameters using for example$ Hansen (1982)'s General Methods of Moments (e.g., Chan et al. (1992)). Discretizationbased methods implicitly assume that more data means more frequent data on a fixed period of observation. This hardly matches the way new data are added to the sample: we typically add today's interest rate at the end of the sample, not the 3 PM interest rate on January 15 , 1992 in between two already existing observations in the sample. Even if such data were available, it is likely that market micro-structure problems, such as the bid-ask spread, the discreteness of the prices observed, and the irregularity of the intra-day sampling interval would complicate considerably the analysis of high frequency data compared to daily or weekly data. Furthermore, identification of the drift is generally impossible on a fixed sampling period, no matter how small the sampling interval. In contrast to this approach we will not require that the sampling interval shrink to obtain asymptotic properties for our estimators.

Our setup has three characteristic features. First, the precise form of the diffusion function of the interest rate process is crucial to price options, as demonstrated by the extensive literature trying to capture a correct specification. Second, it is hard to form an a 
priori idea of the functional form of the diffusion function, as the instantaneous volatility of financial series is not observed. Third, long time-series of daily data on spot interest rates are available. These three elements together, importance of the specification, lack of a priori information regarding that specification, and availability of the data, constitute the perfect setup to try to estimate the instantaneous volatility function nonparametrically. This is the objective of this paper. It is achieved by density-matching: the instantaneous drift and diffusion functions of the short rate process are derived to be consistent with the observed distribution of the discrete data.

The paper is organized as follows. The first part examines the identification and estimation of the drift and diffusion functions of a continuous-time process. Section 2 identifies nonparametrically the diffusion given a restriction on the drift function. Section 3 then constructs the actual estimator, and describes its properties. The second part of the paper uses the results of the first to derive nonparametric option prices for interest rate derivative securities (Section 4). Section 5 computes these prices successively for discount bonds and options on discount bonds. Section 6 concludes. Technical assumptions and details are in Appendix 1-4 while proofs are in Appendix 5.

\section{Nonparametric Identification of the Diffusion Function}

\subsection{Identification via Density-Matching}

When estimating the CAPM, it is known empirically (see Merton (1980)) that the estimates of expected returns tend to have low precision when the observation period is finite, even though the diffusion can be estimated very precisely when the sampling interval is small. For example, suppose that a stock price were to follow $\mathrm{dX}_{\mathrm{t}} / \mathrm{X}_{\mathrm{t}}=\mu \mathrm{dt}+\sigma \mathrm{dW}$ t with $\mu$ and $\sigma$ constant. The maximum-likelihood estimate of $\mu$ from observations between dates 1 and $n$ at interval $\Delta$ is the average of the log-returns, $\hat{\mu}=(1 / n) \sum_{t=1}^{n} \operatorname{Ln}\left(X_{t}\right)$. But $\hat{\mu}=\left(\operatorname{Ln}\left(X_{n}\right)-\operatorname{Ln}\left(X_{1}\right)\right) / n$ is independent of the sampling interval $\Delta$ and cannot be consistent as $\Delta \rightarrow 0$ for fixed $n$. The only hope to identify the drift $\mu$ of the continuous-time process from the data consists in letting the sample period $n$ increase. We will therefore rely on increasing $n$ to derive identification and estimation results.

Identifying without restrictions both the drift and diffusion functions from discretely sampled data is impossible in general. In particular, without further constraints, a pair of functions $\left(\mu, \sigma^{2}\right)$ cannot be distinguished from $\left(a \mu, a \sigma^{2}\right)$ for any constant a, with data discretely sampled at a fixed interval. To identify fully the process, we therefore 
impose a restriction on the form of the drift function so that we can leave the diffusion function unrestricted. Since nothing so far is parametrized, the drift function cannot be restricted simply by fixing some parameter values. Instead, the restriction operates at one extra level of generality. It takes an otherwise completely unrestricted drift function and makes it belong to a smaller class of functions, namely a parametric class.

We will then use an essential property of stochastic differential equations. Consider a discrete normal random variable. Its distribution is obviously entirely characterized by its first two moments, mean and variance. The continuous-time process $r$ in (1.1) in general is not normally distributed. However, because the Brownian increments are Gaussian, it turns out that under regularity conditions an analogous property will hold for stochastic differential equations: the distributions of the process (marginal and transitional densities) are entirely characterized by the first two moments of the process, here the drift and diffusion functions. By the Markov property, it suffices to consider only one set of transitions of the process; longer transitions can always be derived by iterating the shorter transitions.

In particular, the joint parametrizations of $\left(\mu, \sigma^{2}\right)$ adopted in the literature imply specific forms for the marginal and transitional densities of the process. For example, an Ornstein-Uhlenbeck process $\mathrm{dr}_{\mathrm{t}}=\beta\left(\alpha-r_{\mathrm{t}}\right) \mathrm{dt}+\gamma \mathrm{dW}$, generates Gaussian transitional and marginal densities. The stochastic differential equation $d_{t}=\beta\left(\alpha-r_{t}\right) d t+\gamma r_{t}^{1 / 2} d W$ yields a non-central chi-squared transitional distribution while the marginal density is a Gamma distribution (Feller (1951)). The former parametrization for the spot interest rate has been used by Vasicek (1977), while the latter has been derived in a general equilibrium framework by Cox-Ingersoll-Ross $(1985 \mathrm{a}, 1985 \mathrm{~b})$. The functional forms of the transitional densities corresponding to specifications essentially different from these two are not known explicitly (Wong (1964) summarizes the only specifications where they are: linear $\mu$ and quadratic $\sigma^{2}$ ).

Our estimation approach relies on the equivalence between $\left(\mu, \sigma^{2}\right)$ and densities in the other direction: from the densities back to $\left(\mu, \sigma^{2}\right)$. To price derivatives, the drift and diffusion of the short term interest rate process need to be estimated. But they are not observed, nor can they be estimated directly. However the densities of the process can be straightforwardly estimated from data on the short term rate. Therefore instead of specifying parametrically both the drift and diffusion functions and then accepting whatever marginal and transitional densities are implied by these choices --as the various parametric specifications do-- we start with nonparametric estimates of the densities. Then we 
reconstruct the drift and diffusion of the continuous-time process by matching these densities, which we can in turn use for derivative pricing.

Our construction of the pair $\left(\mu, \sigma^{2}\right)$ that will match the densities is the following. Assume that the spot interest rate process $\left\{r_{t}, t \geq 0\right\}$ follows (1.1) under assumptions A1A2 in Appendix 1. These assumptions guarantee the existence and uniqueness of a strong stationary solution to $(1.1)$, on $\mathrm{D}=(0, \infty)$ since nominal interest rates are positive ${ }^{2}$. In particular they rule out the possibility that starting from any interest rate level in $(0, \infty)$ the barriers $\underline{r}=0$ and $\bar{r}=\infty$ could be attained in finite expected time. The drift $\mu(\cdot, \theta)$ depends on an unknown parameter vector $\theta$ while the diffusion $\sigma^{2}(\cdot)$ is an unknown function. The available data consist of realizations of the process sampled at equally spaced discrete dates $0,1, \ldots, n$. The continuous record of observations between each sampling date is unobservable. The asymptotic properties of the estimators as $n \rightarrow \infty$ are derived for an expanding sampling period, i.e., the interest rate is observed over a longer period of time, not by sampling more frequently. The sampling interval is fixed at $\Delta=1$. Let $\pi($.) be the marginal density of the spot rate, and $p\left(\Delta, r_{i+\Delta} \mid r_{t}\right)$ the transition density function between two successive observations.

Consider the Kolmogorov forward equation (e.g., Karlin and Taylor (1981), page 219):

$$
\frac{\partial p\left(\Delta, r_{t+\Delta} \mid r_{t}\right)}{\partial \Delta}=-\frac{\partial}{\partial r_{t+\Delta}}\left(\mu\left(r_{t+\Delta}, \theta\right) p\left(\Delta, r_{t+\Delta} \mid r_{t}\right)\right)+\frac{1}{2} \frac{\partial^{2}}{\partial r_{t+\Delta}^{2}}\left(\sigma^{2}\left(r_{t+\Delta}\right) p\left(\Delta, r_{t+\Delta} \mid r_{t}\right)\right)
$$

In order to construct an estimator of $\sigma^{2}$ from the densities of the process, we use (2.1) to characterize the diffusion function. Note that by stationarity $\int_{0}^{+\infty} p\left(\Delta, r_{t+\Delta} \mid r_{t}\right) \pi\left(r_{t}\right) d r_{t}=\pi\left(r_{t+\Delta}\right)$ has a partial derivative with respect to time equal to zero. Multiply therefore the forward equation (2.1) by the marginal density $\pi\left(r_{1}\right)$ and integrate through the equation with respect to the conditioning variable $r_{t}$ to obtain the ordinary differential equation:

$$
\frac{\mathrm{d}^{2}}{\mathrm{dr}^{2}}\left(\sigma^{2}(\mathrm{r}) \pi(\mathrm{r})\right)=2 \frac{\mathrm{d}}{\mathrm{dr}}(\mu(\mathrm{r}, \theta) \pi(\mathrm{r}))
$$

\footnotetext{
${ }^{2}$ The Vasicek model has the undesirable feature of being distributed on $(-\infty,+\infty)$ as opposed to $(0, \infty)$.
} 
where $r=r_{t+\Delta}$. This equation must be satisfied at any point $r$ in $(0, \infty)$, and at the true parameter value $\theta$. Integrating (2.2) twice with the boundary condition $\pi(0)=0$ now yields:

$$
\sigma^{2}(r)=\frac{2}{\pi(r)} \int_{0}^{r} \mu(u, \theta) \pi(u) d u
$$

This equation shows that once the drift parameter vector $\theta$ has been identified, the diffusion function can be identified from the marginal distribution $\pi($.). The identification of $\theta$ will be based on the transitional distribution. An identifying restriction on the drift is the linear mean-reverting specification $\mu\left(r_{t}, \theta\right) \equiv \beta\left(\alpha-r_{t}\right), \theta \equiv(\alpha, \beta)^{\prime}$. Heuristically, the interest rate is elastically attracted to its equilibrium value $\alpha$ at a speed $\beta \mathrm{dt}$. This is consistent with the parametrization of the drift consistently used in most spot rate models in the literature and will make comparisons with these models possible. The first step consists in deriving:

$$
E\left[r_{t+\Delta} \mid r_{t}\right]=\alpha+e^{-\beta \Delta}\left(r_{1}-\alpha\right)
$$

To see why this must hold, let $\vartheta(r, t)$ be the solution of the backward Kolmogorov equation $\partial \vartheta(r, t) / \partial t=A \vartheta(r, t)$ with initial condition $\vartheta(r, 0)=r$, where $A$ is the backward Kolmogorov operator: $A \vartheta(r, t) \equiv \mu(r, \theta) \partial \vartheta(r, t) / \partial r+(1 / 2) \sigma^{2}(r) \partial^{2} \vartheta(r, t) / \partial r^{2}$. Under assumptions A1-A2, this partial differential equation has the unique solution $\vartheta(r, t)=E\left[r_{t} \mid r_{0}=r\right]$ by Dynkin's formula (e.g., Karlin and Taylor (1981) page 310). Now it is easy to verify directly that the function $\zeta(r, t) \equiv \alpha+e^{-\beta \Delta}(r-\alpha)$ also satisfies the equation with the same initial equation. Thus $\zeta=\vartheta$. Evaluating the equality at $t=\Delta$ gives (2.4).

This procedure can be extended to cover the case of nonlinear restrictions on the drift . In that case the only part of the identification and estimation procedure that has to be modified is (2.4): see Appendix 2. The drift parameters, once identified, can be plugged into (2.3), which remains valid for a generic drift function. We now use (2.4) to identify the parameter vector $\theta$. Ordinary least squares (OLS) clearly identifies the parameters $\gamma$ and $\delta$ in $E\left[r_{t+\Delta}-r_{t} \mid r_{t}\right]=\gamma+\delta r_{t}$, and therefore indirectly $\alpha$ and $\beta$. A discussion of efficiency is provided in the next section. Finally the diffusion function can be completely identified from the joint and marginal density functions $\mathrm{p}(., .1$.$) and \pi($.$) through (2.3)-(2.4). The$ drift is identified from the conditional mean and given the drift the marginal density yields the diffusion function: 
THEOREM 1: Under Assumptions A1-A2, the diffusion function of the underlying spot interest rate process can be identified from its joint and marginal densities by (2.3), where the unknown parameter vector $\theta$ is identified by (2.4).

\subsection{Diffusion for Large and Small Values of the Spot Rate}

The behavior for $\mathrm{r} \rightarrow+\infty$ of the diffusion function given by Theorem 1 is not immediate since the numerator and the denominator of the ratio (2.3) tend both to zero. The resulting effect can nevertheless be characterized by L'Hôpital's Rule as:

$$
\sigma^{2}(r) \cong_{r \rightarrow+\infty} 2 \beta r \pi(r) /\left|\pi^{(1)}(r)\right|
$$

where $\phi(r) \cong_{r \rightarrow+\infty} \varphi(r)$ means $\lim _{\mathrm{r} \rightarrow+\infty} \phi(r) / \varphi(r)=1$ and $\pi^{(1)}$ is the first derivative of $\pi$.

The asymptotic trend of the diffusion function is determined by the speed of decrease of the marginal density $\pi(\cdot)$ to zero: the faster the density goes to zero, the smaller the corresponding diffusion function. Suppose that today's spot rate is high, say $20 \%$. The stationary data on the distribution of the process essentially say that it is very unlikely for the spot rate to be so high. Consider now the spot rate level tomorrow. The increment between today and tomorrow is given by (1.1). If a large level is reached today $\left(\mathrm{r}_{\mathrm{t}}=20 \%\right)$, the mean-reverting drift term will be substantially negative $(\beta>0)$ and drive the process back toward lower levels (say $\alpha=9 \%$ ), which goes in the right direction. If however $\sigma(20 \%)$ happens to be very large then for given realizations of the Brownian increment the stochastic term might compensate the deterministic mean-reverting drift with substantial probability. This would generate a high level of the spot rate which would be incompatible with $\pi($.) very close to zero above $20 \%$.

The link between density and shape of the diffusion function can be made evident by applying successively (2.5) to the parametric family of densities which exhibit an increasingly rapid rate of decrease to zero $(v>1$ and $\omega>0)$ :

$$
\begin{aligned}
& \pi(\mathrm{r}) \equiv \xi \mathrm{r}^{\nu-1} \mathrm{e}^{-\omega r} \text { generates } \sigma^{2}(\mathrm{r}) \cong_{\mathrm{r} \rightarrow+\infty}(2 \beta / \omega) \mathrm{r} \text { (the CIR case) } \\
& \pi(\mathrm{r}) \equiv \xi \mathrm{r}^{\nu-1} \mathrm{e}^{-\omega \mathrm{r}^{2}} \text { generates } \sigma^{2}(\mathrm{r}) \cong_{\mathrm{r} \rightarrow+\infty}(\beta / \omega) \text { (the Gaussian case) } \\
& \pi(\mathrm{r}) \equiv \xi \mathrm{r}^{\nu-1} \mathrm{e}^{-\omega \mathrm{r}^{3}} \text { generates } \sigma^{2}(\mathrm{r}) \cong_{\mathrm{r} \rightarrow+\infty}(2 \beta / 3 \omega)(1 / \mathrm{r}) \text { (faster decay). }
\end{aligned}
$$

The behavior near zero of the diffusion function is also given by L'Hôpital's Rule as $\sigma^{2}(\mathrm{r}) \cong_{\mathrm{r} \rightarrow 0^{+}} 2 \beta \alpha \pi(\mathrm{r}) /\left|\pi^{(1)}(\mathrm{r})\right|$. So for all densities (2.6) we have: $\sigma^{2}(\mathrm{r}) \cong_{\mathrm{r} \rightarrow 0^{+}} 2 \beta \alpha \mathrm{r} /(\nu-1)$. Take densities polynomial in zero $\left(\pi(\mathrm{r}) \cong_{\mathrm{r} \rightarrow 0^{+}} \xi \mathrm{r}^{\nu-1}, v>1\right)$. This encompasses most potential densities since $\pi$ must be zero at zero and smooth. The 
result is then that every density polynomial in zero will yield a diffusion function with the same linear curvature near zero as the square-root process. Thus the CIR parametrization must be a reasonable approximation for small levels of the spot rate. Because the pricing equation for interest rate derivative securities has a strong local character, these securities would tend to be priced correctly by the CIR model when interest rates are low.

\section{Nonparametric Estimation of the Diffusion}

We propose to replace $\theta$ and $\pi($.$) in (2.3) by consistent estimators to obtain an$ estimate of $\sigma^{2}$ (.). Starting with $\pi($.$) , we use the interest rate data \left\{r_{i}, i=1, \ldots, n\right\}$ to form the smooth density estimator $\hat{\pi}(r) \equiv\left(1 / n h_{n}\right) \sum_{i=1}^{n} K\left(\left(r-r_{i}\right) / h_{n}\right)$, based on a kernel function $K\left(\right.$.) and bandwidth $h_{n}$ (see e.g., Silverman (1986) for an introduction to kernel density estimation and Scott (1992) for more details). Regularity conditions on the time-series dependence in the data (Assumption A3), the kernel (Assumption A4) and bandwidth (Assumption A5) are given in Appendix 1. The smooth estimator $\hat{\pi}($.$) can be used in the$ denominator of (2.3) as well as inside the integral, or only in the denominator with the density inside the integral replaced by an empirical density. The two resulting estimators of the diffusion function share the same asymptotic properties.

To estimate $\theta$ consistently, one can simply use OLS in (2.4). The efficiency of the estimator of $\theta$ will not matter for $\sigma^{2}($.), because $\hat{\theta}$ will converge at speed root-n while $\hat{\pi}()$. in the denominator will force the convergence of $\hat{\sigma}^{2}$ (.) to be slower. To obtain nevertheless a better drift estimator than OLS, useful later when pricing derivatives, consider the following procedure. We form the first step OLS estimator of $\theta=(\alpha, \beta)$ ' by a one-to-one transformation from the OLS estimates of $(\gamma, \delta): \alpha=-\gamma / \delta$ and $\beta=-\operatorname{Ln}(1+\delta) / \Delta$. The OLS estimator of $\theta$ can now be plugged along with the kernel estimator $\hat{\pi}($.$) into (2.3) to$ form a nonparametric estimator of $\sigma^{2}$ (.).

Then this diffusion estimator can be used to correct for heteroskedasticity in the residuals from the regression (2.4). We construct the weighting matrix for the second step feasible generalized least squares (FGLS) estimation of $(\gamma, \delta)$ and thus $(\alpha, \beta)$. Note that no serial correlation adjustment is needed in (2.4). Any diffusion process is a Markov process so in particular $E\left[r_{t+\Delta} \mid r_{t}, r_{t-\Delta}\right]=E\left[r_{t+\Delta} \mid r_{t}\right]$. The residuals $\varepsilon_{t+\Delta} \equiv r_{t+\Delta}-\left(\gamma+(1+\delta) r_{t}\right)$ from the regression $E\left[r_{t+\Delta}-r_{t} \mid r_{t}\right]=\gamma+\delta r_{t}$ are therefore conditionally uncorrelated, that is: $\mathrm{E}\left[\varepsilon_{\mathrm{t}+\Delta} \varepsilon_{\mathrm{t}} \mid \mathrm{r}_{\mathrm{t}}, \mathrm{r}_{\mathrm{t}-\Delta}\right]=0$. If the interest rate data were independent and identically distributed, this procedure would achieve the semiparametric efficiency bound of Chamberlain (1987) (see also Robinson (1987) for a two-stage setup). To our knowledge, there exists no 
known efficiency bound for dependent data. The following result details the asymptotic properties of the diffusion estimator:

THEOREM 2: $\quad$ Under assumptions A1-A5:

(i) The estimator $\hat{\sigma}^{2}($.$) is pointwise consistent and asymptotically normal:$ $h_{n}^{1 / 2} n^{1 / 2}\left\{\hat{\sigma}^{2}(r)-\sigma^{2}(r)\right\} \stackrel{d}{\longrightarrow} N\left(0, V_{\sigma^{2}}(r)\right)$, with asymptotic variance:

$$
V_{\sigma^{2}}(r)=\left\{\int_{-\infty}^{+\infty} K(u)^{2} d u\right\} \sigma^{4}(r) / \pi(r)
$$

(ii) The asymptotic variance $V_{\sigma^{2}}(r)$ can be consistently estimated by:

$$
\hat{V}_{\sigma^{2}}(r)=\left\{\int_{-\infty}^{+\infty} K(u)^{2} d u\right\} \hat{\sigma}^{4}(r) / \hat{\pi}(r) .
$$

(iii) At different points $r$ and $r^{\prime}$ in $(0, \infty), \hat{\sigma}^{2}(r)$ and $\hat{\sigma}^{2}\left(r^{\prime}\right)$ are asymptotically independent.

The intuition behind the result is the following. There are three elements pluggedinto the right hand side of (2.3): $\hat{\theta}, \hat{\pi}($.$) inside the integral and \hat{\pi}($.$) in the denominator.$ The first two are responsible for root-n terms, while the third generates a term converging at the slower speed root-bandwidth times root-n. The fast terms can be considered as fixed when computing the asymptotic distribution. Only the slowest term will matter. Part (iii) of the theorem is typical of pointwise kernel estimators (see Robinson (1983)) and is useful to know for inference purposes. The consistent estimator of the pointwise asymptotic variance makes it possible to construct pointwise confidence intervals, or later obtain estimates of the variance of derivative security prices. Global confidence intervals for $\sigma^{2}($.) are derived and used for specification testing in a related paper (Aït-Sahalia (1994)).

\section{Pricing Interest-Rate-Derivative Securities}

\subsection{Nonparametric Plug-In}

Given the underlying spot interest rate process $\left\{r_{t} / t \geq 0\right\}$ described by a stochastic differential equation of the type (1.1), standard arbitrage arguments (as for example in Vasicek (1977), Section 3) determine the price $U(r, t)$ of any interest-rate-derivative security with time-to-maturity $t$ and maturity date $T$ when $r$ is the spot interest rate . Let $\lambda(r)$ be the risk premium factor or market price of interest rate risk --which must be the same for all securities-- $c(r, t)$ the cash flow rate paid by the security per unit of time and $g(r)$ the 
payoff of the derivative security at maturity. $U(r, t)$ satisfies the following partial differential equation:

$$
\mathrm{LU}(\mathrm{r}, \mathrm{t})=-\mathrm{c}(\mathrm{r}, \mathrm{t})
$$

where $L$ is the parabolic differential operator:

$$
L U \equiv-\partial U / \partial t+\left\{\sigma^{2}(r) / 2\right\}\left(\partial^{2} U / \partial r^{2}\right)+\{\mu(r, \theta)-\lambda(r) \sigma(r)\}(\partial U / \partial r)-r U
$$

This is a Cauchy problem with initial condition $U(r, 0)=g(r)$. The continuous payment rate $c(.,$.$) , the payoff g($.$) at maturity as well boundary condition(s) depend on the$ particular security considered. Assumptions A6-A8 in Appendix 3 impose regularity conditions. Consider first a discount bond with face value equal to $\$ 100$ and maturing at date $T$. Let $U(r, t) \equiv B(r, t, T)$ be its price at date (T-t) when the spot interest rate is $r$. This case corresponds to:

(4.3) $c(r, t)=0$ (zero-coupon), $g(r)=100$ for all $r \geq 0$ (initial condition), $\lim _{r \rightarrow \infty} U(r, t)=0$ for all $t \geq 0$ (boundary condition).

Consider next a call option ${ }^{3}$ on a discount bond. The call option expires at date $\mathrm{T}$, has an exercise (or strike) price $X$, and the underlying discount bond matures at date $S$ where $T \leq S$. Let $U(r, t) \equiv C(r, t, T ; s, X)$ be the call price at time-to-maturity $t$ when the spot interest rate is $r$. Then:

$$
c(r, t)=0, g(r)=\max (0, B(r, S-T, S)-X) \text { for all } r \geq 0, \lim _{r \rightarrow \infty} U(r, t)=0 \text { for all }
$$
$\mathrm{t} \geq 0$.

Other examples can be treated similarly. A coupon bond would be identical to (4.3), except that $\mathrm{c}$ is then the coupon rate received at $t$. An interest rate swap of $r$ against an exogenous rate $r^{\prime}$ can be viewed as a contract paying at rate $c=r-r^{\prime}$. A cap (resp. floor) is a loan at variable rate that is guaranteed to be less (resp. more) than some level $\overline{\mathrm{r}}$ (resp. $\underline{r}$ ); it can be seen as a derivative with $c=\min (r, \bar{r})(\operatorname{resp} . c=\max (r, \underline{r}))$. In all these cases $\mathrm{g}$ is the constant face value of the contract. A yield curve call option struck at $X$ has $c=0$ and $g(r)=\max (0, Y(n, T+n, r)-X)$ where $Y(n, T+n, r)$ is the yield-to-maturity at date $T$ of a $n$-year bond. A binary yield option has $c=0$ and $g(r)=1(Y(n, T+n, r) \geq X)$. A power-p yield option

3 It is never optimal to exercise early an American call because the underlying bond pays no coupon, so American and European calls on discount bonds have the same value. 
has $c=0$ and $g(r)=Y(n, T+n, r)^{p}$. A yield curve slope call option has $c=0$ and $g(r)=\max (0,(Y(n, T+n, r)-Y(m, T+m, r)) /(n-m)-X)$, for some $n>m$.

When $\sigma(r)=\sigma$ and $\lambda(r, t)=\lambda$ are constant, (4.1)-(4.3) and (4.1)-(4.4) have known solutions (Vasicek (1977) and Jamshidian (1989) respectively). When $\sigma(r)=\sigma r^{1 / 2}$ and $\lambda(r, t)=\lambda r^{1 / 2} / \sigma,(4.1)-(4.3)$ and (4.1)-(4.4) also have known solutions (CoxIngersoll-Ross (1985b)). As was discussed earlier, these closed-form solutions all assume a particular parametrization of the diffusion term of the underlying spot interest rate. Since the assumptions are different, the pricing formulas differ. Hull and White (1990) compared them for a set of arbitrary values of the parameters (not estimated from actual data and timedependent) and found that the formulas can differ by as much as $15 \%$ (see their Table 1).

We estimate derivative prices by solving the pricing partial differential equation with the drift parameters replaced by the estimates $\hat{\alpha}$ and $\hat{\beta}$ and the unknown function $\sigma^{2}($.) by its nonparametric estimator $\hat{\sigma}^{2}($.) described in Section 3 . We specify that the market price of interest rate risk is constant ${ }^{4}$ and estimate it by minimizing the squared deviations between a given yield curve and that implied by the model. The only justification for the assumptions leading to $\lambda(\mathrm{r}, \mathrm{t})=\lambda_{\mathrm{VAS}}$ or $\lambda(\mathrm{r}, \mathrm{t})=\lambda_{\mathrm{CIR}} \mathrm{r}^{1 / 2} / \sigma_{\mathrm{CIR}}$ in the Vasicek and CIR models respectively is that they yield explicit solutions given their respective choices of drift and diffusion. Here any other function for the market price of risk could be used.

\subsection{The Asymptotic Distribution of Nonparametric Prices}

We show next that derivative prices $\hat{U}$ still converge at the usual parametric speed root-n. This holds even though the $\sigma^{2}$ function is estimated non-parametrically and converges slower than root-n (see Theorem 2). The intuition for the result is as follows. Consider the ordinary differential equation: $d U(r) / d r=\sigma^{2}(r) U(r)$ with initial condition $U(0)=U_{0}$. Its solution is simply: $U(r)=U_{0} \exp \left(\int_{0}^{r} \sigma^{2}(x) d x\right)$. Suppose now that the coefficient function $\sigma^{2}$ (.) of the differential equation were unknown and estimated nonparametrically by $\hat{\sigma}^{2}\left(\right.$.) at rate $n^{1 / 2} h_{n}^{1 / 2}$. Estimate the solution $U($.$) by$ $\hat{U}(r)=U_{0} \exp \left(\int_{0}^{r} \hat{\sigma}^{2}(x) d x\right)$. Now the integral $\int_{0}^{r} \hat{\sigma}^{2}(x) d x$ will converge at rate $n^{1 / 2}$, and so will the estimator $\hat{U}(r)$. A similar example would have the derivative price $U$ depend

${ }^{4}$ Learning more about the specification of the market price of risk would require data on cross-sections of derivatives. The market price of risk could potentially be estimated nonparametrically as well by fitting exactly a price curve, along the lines of the "arbitrage-free" literature on interest rate derivatives. 
upon a nonparametric estimate of the cumulative distribution function (converging at rate $n^{1 / 2}$ ) as opposed to the density (converging at rate $n^{1 / 2} h_{n}^{1 / 2}$ ).

The partial differential equation (4.1) satisfied by derivative prices is obviously substantially more complex than the simple ordinary differential equation above. However it shares the two main insights of that example. First, the solution is also characterized as an integral over the coefficient functions. Second, the coefficient functions are similarly estimated at rate $\mathrm{n}^{1 / 2} \mathrm{~h}_{\mathrm{n}}^{1 / 2}$ (the diffusion function $\left.\sigma^{2}\right)$ or faster $\left(\mathrm{n}^{1 / 2}\right.$ for all the parameters $\alpha, \beta$ and $\lambda$ ). Given this intuition, the first step of the proof is to obtain a representation of derivative prices as an integral over the diffusion estimator. We consider the fundamental solution $\Gamma(\mathrm{r}, \mathrm{t} ; \mathrm{x})$ of $(4.1)^{5}$. This is the solution for $\mathrm{x}$ fixed in $(0, \infty)$ of the Cauchy problem $L \Gamma(r, t ; x)=0$ with initial condition $\Gamma(r, 0 ; x)=\delta_{(x)}(r)$ (a mass point at $x$ ). The following lemma will provide the integral characterization needed to obtain the asymptotic distribution of the derivative prices:

LEMMA 1: Under assumptions A6-A8, there exists a unique solution on $(0, \infty) x(0, T]$ to the pricing partial differential equation, and it has the form:

$$
U(r, t)=\int_{0}^{+\infty} \Gamma(r, 0 ; x) g(x) d x+\int_{0}^{t} \int_{0}^{+\infty} \Gamma(r, \tau ; x) c(x, \tau) d x d \tau .
$$

The fundamental solution $\Gamma(r, t ; x)$ is twice-differentiable in $r$ and $x$, once in $t$, and is a twice-differentiable function of $\sigma^{2}(x)$. It does not depend on $\sigma^{2}(r)$.

The dependence of the fundamental solution $\Gamma(\mathrm{r}, \mathrm{t} ; \mathrm{x})$ on $\sigma^{2}(\mathrm{x})$ is detailed in the proof of Lemma 1 . As a result $U(r, t)$ will depend on $\sigma^{2}$ only through integrals of functions of $\sigma^{2}(x) d x$, which converge at rate $n^{1 / 2}$. Therefore the speed of convergence of the derivative prices will not be slowed down by the use of a nonparametric estimator of $\sigma^{2}$. The benefits from using a nonparametric estimator of $\sigma^{2}$ can be substantial in terms of avoiding misspecification of the derivative prices. This gain in robustness is achieved at no cost in terms of convergence speed for the prices:

THEOREM 3: $\quad$ Under assumptions A1-A9 the nonparametric price of a derivative security satisfies:

\footnotetext{
${ }^{5}$ For example, the transition density of the process over finite intervals of length $\Delta, p\left(\Delta, r_{1+\Delta} \mid r_{1}\right)$, is the fundamental solution $\Gamma$ corresponding to the backward Kolmogorov operator $-\partial U / \partial t+A U$ (i.e., the same as $L$ in (4.2) but with $\lambda=0$ ). If $\mu$ and $\sigma$ were parametrized in (1.1) then the parameters could be estimated by maximum-likelihood: solve numerically for $\Gamma$ this partial differential equation to obtain the transition density of the model. By the Markov property, this gives the likelihood function.
} 


$$
n^{1 / 2}\{\hat{U}(r, t)-U(r, t)\} \stackrel{d}{\longrightarrow} N(0, V(r, t))
$$

with asymptotic variance $V(r, t)=\operatorname{VAR}\left(u_{(r, t)}\left(x_{t}\right)\right)+2 \sum_{k=1}^{\infty} \operatorname{COV}\left(u_{(r, t)}\left(x_{t}\right), u_{(r, t)}\left(x_{t+k}\right)\right)$, where $\mathrm{x}_{\mathrm{t}} \equiv\left(\mathrm{r}_{\mathrm{t}}, \mathrm{r}_{\mathrm{t}+\Delta}\right)$ and $u_{(r, t)}$ is a cadlag function ${ }^{6}$.

To implement Theorem 3 in practice one needs the addition of a consistent estimator of the asymptotic variance $V(r, t)$. Since the expression for $u_{(r, t)}$ is complicated and $V(r, t)$ depends upon the entire serial correlation structure of the interest rate data, the bootstrap technique can be used in practice. The validity of the bootstrap estimator of $V(r, t)$ in this context is proved in Ait-Sahalia (1992). The estimator consists in two simple steps?:

(1) Redraw from the original spot interest rate data. The resampling procedure redraws from blocks of contiguous observations to preserve the serial correlation existing in the original data, an idea introduced by Künsch (1989) and Liu and Singh (1992).

(2) Next estimate the drift and diffusion function associated with this new dataset as in Section 3 and then estimate the market price of risk and compute the resulting bond prices $\hat{U}^{*}(r, t)$. The bootstrap estimator $\hat{V}^{*}(r, t)$ of $V(r, t)$ is the sample variance of the difference of bond prices $\hat{U}^{*}(r, t)-\hat{U}(r, t)$.

Finally, instead of solving (4.1), derivative prices could have been computed by Monte-Carlo simulations of the sample paths of the risk-neutral process $\mathrm{dr}_{\mathrm{t}}=\left\{\mu\left(\mathrm{r}_{\mathrm{t}}, \theta\right)-\lambda\left(\mathrm{r}_{\mathrm{t}}\right) \sigma\left(\mathrm{r}_{\mathrm{t}}\right)\right\} \mathrm{dt}+\sigma\left(\mathrm{r}_{\mathrm{t}}\right) \mathrm{dW}$. The sample paths, all starting at $\mathrm{r}$ at date T-t and finishing at date $\mathrm{T}$, would be simulated with the risk-neutral drift and diffusion replaced by their estimates. Rewriting (4.5) as the Feynman-Kac conditional expectation under the riskneutral dynamics gives the prices:

$$
U(r, t)=E\left[g\left(r_{T}\right) \exp \left\{-\int_{T-t}^{T} r_{u} d u\right\}+\int_{T-t}^{T} \exp \left\{-\int_{T-t}^{\tau} r_{u} d u\right\} c\left(r_{\tau}, \tau\right) d \tau \mid r_{T-t}=r\right] .
$$

The price $U(r, t)$ can then be estimated by averaging the argument of the conditional expectation over the simulated sample paths.

\footnotetext{
${ }^{6}$ Cadlag $=$ Right-continuous-left-limit.

7 In terms of computer programming, the computation of the bootstrap standard errors requires only the addition of a loop for the resampling scheme.
} 


\section{Discount Bond and Bond Option Pricing}

\subsection{The Data}

The spot rate used is the seven-day Eurodollar deposit rate, bid-ask midpoint, from Bank of America. The data are daily from June 1, 1973 to February 25, 1995. Interest rates paid on other short-term financial assets such as commercial paper or T-Bills typically move closely with the Eurodollar rate. A time-series plot of the data is provided in Figure 1. Choosing a seven-day rate --such as the seven-day Eurodollar-- as the underlying factor for pricing derivatives is a necessary compromise between: (i) literally taking an "instantaneous" rate and (ii) avoiding some of the spurious microstructure effects associated with overnight rates. For example, the second Wednesday settlement effect in the Federal Funds market creates a spike in the raw Federal Funds data that would have to be smoothed.

The rates quoted are originally bond-equivalent yields. They were transformed to continuously-compounded yield-to-maturity, deriving the current price B of the instrument from $r_{B E Y}=((100-B) / B)(365 /(T-t))$ and then computing the continuously compounded yield-to-maturity from $B=100 \exp \left(-r_{\text {YтM }}(T-t)\right)$. Monday is taken as the first day after Friday. Whereas weekend effects have been documented extensively for stock prices, there does not seem to be a conclusive weekend effect in money market instruments. Descriptive statistics are provided in Table 2. Since the autocorrelations of the interest rate levels decay slowly, Table 2 also reports the results of an augmented Dickey-Fuller nonstationarity test. The nonstationarity hypothesis is rejected at the $90 \%$ level. The test is known to have low power, so even a slight ejection means that stationarity of the series is likely.

Four other daily interest rate series have also been used to check the robustness of the results. First the overnight Federal Funds rate (source: Telerate). Second the one-month London Eurodollar deposit rate (average LIBOR bid-ask, source: Bank of America and Reuters). Third the one and three-month continuously-compounded bond-equivalent yields on Treasury bills (source: Bank of America). In general, the coefficient $\beta$ of meanreversion is lower when estimated on a longer-maturity proxy for the spot rate (e.g., estimated on three-month T-Bills vs. seven-day Eurodollar vs. Federal Funds). Also, longer maturity proxies are less volatile. Overall, all five series produce qualitatively similar shapes for $\sigma^{2}$. 


\subsection{Nonparametric Diffusion Estimation}

\subsubsection{Drift Estimation}

The first step consists in estimating the drift coefficients $\alpha$ and $\beta$ by OLS. Drift estimates are reported in Table 3 for daily sampling of the annualized spot rate. These estimates will be used to construct the nonparametric diffusion estimator. Given the diffusion estimator, the second-step semiparametric FGLS estimates of the drift are computed. For both stages of drift estimates, the speed of mean reversion $\beta$ is slow.

For comparison purposes, generalized method of moments (GMM) estimates of the Vasicek and CIR models are in Table 3. The GMM estimates of $\alpha, \beta$ and $\sigma^{2}$ for the Vasicek and CIR models are obtained from the following four moment conditions ( $\Delta=1$ day):

$$
f_{t}(\theta) \equiv\left[\varepsilon_{t+\Delta}, \varepsilon_{t+\Delta} r_{t}, \varepsilon_{t+\Delta}^{2}-E\left[\varepsilon_{t+\Delta}^{2} \mid r_{t}\right],\left(\varepsilon_{t+\Delta}^{2}-E\left[\varepsilon_{t+\Delta}^{2} \mid r_{t}\right]_{t}\right) r_{t}\right]
$$

where $\varepsilon_{t+\Delta} \equiv\left(r_{t+\Delta}-r_{t}\right)-E\left[\left(r_{t+\Delta}-r_{t}\right) \mid r_{t}\right]$ with $E\left[r_{t+\Delta}-r_{t} \mid r_{1}\right]=\left(1-e^{-\beta \Delta}\right)\left(\alpha-r_{t}\right)$ for both models. The exact conditional variance of interest rate changes over time intervals of length $\Delta$ is given by $E\left[\varepsilon_{t+\Delta}^{2} \mid r_{t}\right]=V\left[r_{t+\Delta} \mid r_{t}\right]:$

$$
\left\{\begin{array}{l}
\text { Vasicek: } \quad \mathrm{E}\left[\varepsilon_{\mathrm{t}+\Delta}^{2} \mid \mathrm{r}_{\mathrm{t}}\right]=\left(\sigma^{2} / 2 \beta\right)\left(1-\mathrm{e}^{-2 \beta \Delta}\right) \\
\text { CIR: } \quad \mathrm{E}\left[\varepsilon_{t+\Delta}^{2} \mid \mathrm{r}_{\mathrm{t}}\right]=\left(\sigma^{2} / \beta\right)\left(\mathrm{e}^{-\beta \Delta}-\mathrm{e}^{-2 \beta \Delta}\right) \mathrm{r}_{\mathrm{t}}+\left(\sigma^{2} / 2 \beta\right)\left(1-\mathrm{e}^{-\beta \Delta}\right)^{2} \alpha
\end{array}\right.
$$

These moments correspond to transitions of length $\Delta$ and are not subject to discretization bias. Since these GMM systems are overidentified, we weighted the criterion optimally (see Hansen (1982)). Because of overidentification, the first two moments do not reduce to OLS in Table 3.

\subsubsection{Marginal Density Estimation}

The nonparametric kernel estimator of the density is reported in Figure 2. The kernel and optimal bandwidth are described in Appendix 1. Figure 2 also reports the Gaussian and Gamma densities corresponding to the Vasicek and CIR processes (using the respective GMM estimates of their parameters). Noticeable features of the nonparametric density estimator include its fat tail (most high observations were recorded in the two years after the 1979 inflexion of the monetary policy). This feature is less accentuated when the subperiod 1908-82 is not included in the sample. Whether this subperiod should ultimately be included in the sample depends upon one's view regarding the likelihood that high 
interest rates occur again in the future. Under stationarity of the spot rate process, this subperiod provides valuable information on what can potentially happen to the spot rate. Alternatively, a model allowing for multiple interest rate regimes might be considered appropriate.

The density also exhibits a very fast decay to zero above $20 \%$, due to the fact that in the sample very few interest rate levels are recorded outside this range and that the extreme observations are clustered next to the absolute smallest and largest ones (as opposed to being slowly spread on both ends of the density support). Compared to the Vasicek and CIR densities, the nonparametric density exhibits more variation and a combination of a longer tail and a faster rate of decay for large values of the interest rate. These differences will naturally be reflected in the diffusion function. A $99 \%$ pointwise confidence band for the nonparametric density is plotted in Figure 3, using the classical asymptotic distribution of the kernel density estimator. The large sample size makes it possible to estimate the density very precisely.

\subsubsection{Diffusion Estimation}

Given the drift parameters and nonparametric density estimator already constructed, the method of Section 3 yields the nonparametric estimator of the diffusion function, reported in Figure 4. The first noteworthy aspect of the graph is that $\sigma^{2}$ is globally an increasing function of the level of the interest rate between 0 and 14\%. This gives credence to the widely expected result that high interest rates should vary more than low interest rates. Above $14 \%$ however, the diffusion function flattens and then decreases.

At high interest rates the drift in the process (2.1) pulls the process back toward the level of $\alpha=9 \%$. The fact that the density translates into a diffusion function with this shape depends upon the specific strength of the mean reversion effect in the drift. For example, the specification of a stronger mean-reverting drift, such as any power of the current specification, e.g., $\mu(r, \theta) \equiv \alpha_{0}+\alpha_{1} r+\alpha_{2} r^{2}+\alpha_{3} / r$, would make the process "more able" to pull back the spot rate toward $9 \%$ from a high level even in the presence of a larger variance. A stronger drift (taking large negative values at high levels of $r$ ) would compensate for that higher potential variation and tilt the transitional density toward the left as does a medium-strength drift (e.g., linear) combined with a low diffusion. It is easy to extend the discussion in Section 2.2 to see the balancing effects of a stronger (resp. weaker) mean-reverting drift with a higher (resp. lower) diffusion.

At any rate, if a model insists on specifying a linear drift $\mu(r, \theta) \equiv \beta(r-\alpha)$ as do the Vasicek, CIR and most other models, then the diffusion function compatible with the 
data is given by Figure 4 and looks neither linear nor flat. A formal test of misspecification of these parametrizations can be found in Ait-Sahalia (1994). A linear specification for the diffusion (CIR) is a relatively good approximation only on 0 to $9 \%$. This is not too surprising since it was proved in Section 2.2 that any density polynomial in zero would give rise to a diffusion function linear for small values of the interest rate. The nonparametrically estimated $\sigma^{2}$ function increases faster than $\mathrm{r}$ for medium values of the interest rate ( 9 to $14 \%$ ), flattens (14 to 17\%), then decreases (above 17\%). The CIR diffusion instead increases steadily as a linear function of $r$.

Figure 5 reports pointwise confidence intervals for the nonparametric diffusion estimator. This asymptotic distribution given by Theorem 2 has the feature that the diffusion function is estimated more precisely in highly populated interest rate regions. Conversely, the confidence band tends to increase above $14 \%$ reflecting the relative scarcity of the data. The estimates of $\sigma^{2}$ are stable across subperiods of the sample, with the exception that subperiods which do not include the high interest rate years 1980-82 produce estimates of $\pi$ with a truncated right tail, which in turn shift the graph of $\sigma^{2}$ to the left, and have a lower overall level of volatility.

\subsection{The Term Structure of Interest Rates}

The market price of risk is estimated for each model by minimizing the sum of squared deviations across maturities between a given target yield curve and the yields produced by the respective model. To obtain a realistic target yield curve, we take the average yield curve over the sample period obtained from the end-of-month Reuters data. The yield curve for coupon bonds is transformed to a discount yield curve by using the standard technique of fitting third-order polynomial splines. From now on we treat this yield curve as fixed.

The target yield curve is plotted in Figure 6. Market price of risk estimates are in Table 4. Table 5 details the bond prices computed under the CIR, Vasicek and nonparametric models. Standard errors for the nonparametric prices, derived as indicated in Section 4, are also reported. As a result of fitting a common yield curve the CIR and Vasicek prices are generally within one standard deviation of the nonparametric prices. By eliminating differences in the prices of the underlying bonds, differences in option prices can be attributed to differences in the volatility of the underlying bonds, which is the determining factor. 


\subsection{Bond Option Pricing}

Table 6 reports call option prices on a 5-year discount bond and the nonparametric prices standard errors. Option prices reflect mostly differences in the second moment of the spot rate, where differences across models are more pronounced. By Itô's Lemma, the risk-neutral bond price follows $\mathrm{dB} / \mathrm{B}=\mathrm{rdt}+\{\sigma(\mathrm{r})(\partial \mathrm{B} / \partial \mathrm{r}) / \mathrm{B}\} \mathrm{dZ}$ when the risk-neutral spot rate has dynamics: $\mathrm{dr}=\{\mu(\mathrm{r})-\lambda(\mathrm{r}) \sigma(\mathrm{r})\} \mathrm{dt}+\sigma(\mathrm{r}) \mathrm{dZ}$. Figure 7 reports the volatilities $\sigma(\mathrm{r})(\partial \mathrm{B} / \partial \mathrm{r}) / \mathrm{B}$ of a 5-year bond price as a function of the spot rate level $r$. How large the bond volatility is under each model provides some intuition for the relative magnitude of the three prices in Table 6.

When the spot rate is $2 \%$ we see that the bond price volatility from the Vasicek model is larger than that from the CIR and nonparametric models, which are close to each other. As a result, the Vasicek option prices are larger than the other two, and significantly different from the nonparametric prices. Not surprisingly this effect is more visible for deep-out-of-the-money options (the right column of Table 6), and shorter maturity options (the upper rows in both parts of Table 6). Intuitively, the shorter the option the more its price depends on the values of the parameters evaluated close to the current value of the spot rate $r_{t}$. Similarly when the short rate is $14 \%$, the bond price volatility from the nonparametric spot process is higher than CIR, which in turn is higher than Vasicek. The ordering of the option prices is modified accordingly. Both the Vasicek and CIR prices then fall outside one or more standard deviations of the nonparametric prices.

\section{Conclusions}

This paper has proposed a procedure to estimate stochastic models while preserving their basic continuous-time structure. Properties of stochastic differential equations were exploited to both identify and estimate the drift and diffusion function of the process of interest. In particular, it was shown that the entire diffusion function can be estimated nonparametrically once a parametrization of the drift is adopted. The density-matching procedure starts from estimates of the densities of the process (which could as well be parametric) and then recovers the drift and the diffusion functions that are consistent with the density estimates. Asymptotic distributions are computed for the diffusion function of the underlying asset process and for the prices of the derivative securities. Even though the diffusion is estimated nonparametrically, and therefore converges slower than root- $n$, the speed of convergence of the derivative prices is not penalized. 
The general density-matching approach could potentially be applied to a wide variety of processes in economics and finance, when it is important to identify precisely the instantaneous variance, using discrete data without discretizing the continuous-time process. The pricing of interest-rate derivative securities was precisely such a problem, since option prices depend on the particular shape of the diffusion function of the underlying spot interest rate. With a linear mean-reverting drift, consistent with most of the literature, the diffusion function was found to be increasing linearly, then exponentially, before flattening and decreasing. This was shown to have implications for the pricing of derivative securities. Other relevant applications can be considered in the future. The method can easily be extended to cover multi-dimensional stochastic differential equations and therefore multi-factor models of the term structure. Hedging strategies in the option market can be assessed with the help of the nonparametric prices. A similar estimation approach for the drift and diffusion could be implemented to estimate the process followed by stock returns, or exchange rates to price foreign currency options, or identify the consequences of target zone bands.

Graduate School of Business, University of Chicago, 1101 East 58th Street, Chicago, IL 60637-1561, U.S.A., and National Bureau of Economic Research.

July 1992. Revised July 1995. 


\section{APPENDIX}

\section{Appendix 1: Regularity Conditions for the Nonparametric Estimation of the Diffusion}

\section{ASSUMPTION A1:}

(i) The drift and diffusion functions have $s \geq 2$ continuous derivatives on $(0, \infty)$.

(ii) $\sigma^{2}>0$ on $(0, \infty)$.

(iii) The integral of $m(v) \equiv\left(1 / \sigma^{2}(v, \theta)\right) \exp \left\{-\int_{v}^{\bar{\varepsilon}}\left[2 \mu(u, \theta) / \sigma^{2}(u, \theta)\right] d u\right\}$, the speed measure, converges at both boundaries of $(0, \infty)$.

(iv) The integral of $s(v) \equiv \exp \left\{\int_{v}^{\bar{\varepsilon}}\left[2 \mu(u, \theta) / \sigma^{2}(u, \theta)\right] d u\right\}$, the scale measure, diverges at both boundaries of $(0, \infty)$.

In Al(iii)-(iv), $\bar{\varepsilon}$ is fixed in $(0, \infty)$ and its particular choice is irrelevant. Under Al (i)-(ii) the stochastic differential equation (1.1) admits a unique strong solution. Note that by the mean-value theorem A1(i) implies the local Lipschitz and growth conditions:

(LLG) For each compact subset of $\mathrm{D}=(0, \infty)$ of the form $K \equiv[1 / R, R], R>0$, there exist constants $C_{1}$ and $C_{2}$ such that for all $x$ and $y$ in $K$ :

$$
\left\{\begin{array}{l}
|\mu(\mathrm{x})-\mu(\mathrm{y})|+|\sigma(\mathrm{x})-\sigma(\mathrm{y})| \leq \mathrm{C}_{1}|\mathrm{x}-\mathrm{y}| \\
|\mu(\mathrm{x})|+|\sigma(\mathrm{x})| \leq \mathrm{C}_{2}\{1+|\mathrm{x}|\}
\end{array}\right.
$$

Global Lipschitz and growth conditions are usually imposed on the drift and diffusion functions ${ }^{8}$ to guarantee existence and uniqueness of a strong solution to the stochastic differential equation (1.1). These global conditions fail to be satisfied for many models of interest here. It is known that the local Lipschitz condition is sufficient for pathwise uniqueness of the solution (Itô's Theorem, see e.g., Karatzas and Shreve (1991), Theorem 5.2.5 page 287). Now A1(i)-(ii) guarantees the existence of a weak solution (see

8 Global means here that the same constants $C_{1}$ and $C_{2}$ must be valid on the entire domain $D$ of the diffusion. For a statement of Itô's classical result with global conditions, see e.g., $\varnothing \mathrm{ksendal}$ [1992], Theorem 5.5, or Karatzas and Shreve [1991], Theorem 5.2.5 page 287. Even the weaker Yamada-Watanabe condition (see Karatzas and Shreve [1991], 5.2.13 page 291) fails to be satisfied for interesting economic models. Both would imply existence and uniqueness of a strong solution to (1.1). 
e.g., Karatzas and Shreve (1991), Theorem 5.5.15 page 341), up possibly to an explosion time. Then the existence of a weak solution combined with pathwise uniqueness imply strong existence (a corollary to the Yamada and Watanabe Theorem, e.g., Karatzas and Shreve (1991), Proposition 5.3.20 page 309-310). Therefore A1(i)-(ii) imply that (1.1) admits a unique strong solution up possibly to an explosion time.

The role of Al(iii)-(iv) is to rule out explosions in finite expected time and guarantee stationarity of the process. The solution is a Markov process with timehomogenous transition densities (i.e., such that transition probability density $p(s, y ; t, x)$ from $x$ at time $t$ to $y$ at time s depends only on $t-s$, not on $t$ and s separately). Al(iv) further guarantees that starting from any point in the interior of the state space $D=(0, \infty)$, the boundaries $\underline{x}=0$ and $\bar{x}=+\infty$ cannot be attained in finite expected time. Indeed define the scale measure $S(\varepsilon, \bar{\varepsilon}) \equiv \int_{\varepsilon}^{\bar{\varepsilon}} \mathrm{s}(\mathrm{v}) \mathrm{dv}$. Zero cannot be attained in finite time by the spot rate if $\lim _{\varepsilon \rightarrow 0} S(\varepsilon, \bar{\varepsilon})=+\infty$ (see Karlin and Taylor (1981), Lemma 15.6.3 and Table 15.6.2). Infinity cannot be attained in finite time if: $\lim _{R \rightarrow+\infty} S(\bar{\varepsilon}, R)=+\infty$.

An alternative condition can be formulated in terms of Lyapunov functions (see Hasminskii (1980)): Assume that there exists a twice continuously differentiable nonnegative function $\vartheta$ on $D$ such that $\mu(x, \theta) \partial \vartheta(x) / \partial x+(1 / 2) \sigma^{2}(x) \partial^{2} \vartheta(x) / \partial x^{2} \leq-1$ for all $x$ in $D=(0,+\infty)$ outside a compact interval of the form $C \equiv[1 / R, R], R>0$. Also assume that $\lim _{x \rightarrow 0} \vartheta(x)=\lim _{x \rightarrow \infty} \vartheta(x)=\infty$. Since $\vartheta \geq 0$, this condition implies the weaker inequality: $\mu(x, \theta) \partial \vartheta(x) / \partial x+(1 / 2) \sigma^{2}(x) \partial^{2} \vartheta(x) / \partial x^{2} \leq c \vartheta(x)$ for some (in fact any) constant $c \geq 0$. Under this weaker inequality, we can only be sure to obtain a Markov solution with time-homogenous transition densities (Hasminskii (1980), Theorem III.4.1 and Remark III.4.1). This is necessary but not sufficient for the process to be stationary: for example a Brownian motion has time-homogenous transition densities (Gaussian) but is obviously not a stationary process. For our estimation strategy to work, we need the process solution to (1.1) to be stationary and therefore impose in Al(iii) the stronger requirement that $\mu(\mathrm{x}, \theta) \partial \vartheta(\mathrm{x}) / \partial \mathrm{x}+(1 / 2) \sigma^{2}(\mathrm{x}) \partial^{2} \vartheta(\mathrm{x}) / \partial \mathrm{x}^{2} \leq-1$ (use Theorem III.5.1 and footnote 9 in Hasminskii (1980), or see directly that this condition implies the existence of an integrable solution $\pi$ to (2.2) for given drift and diffusion functions). It then follows that the stationary marginal density $\pi$ exists (and integrates to one by construction) and is continuously differentiable on D up to order $s \geq 2$.

ASSUMPTION A2: The stationary density $\pi$ is strictly positive on $(0, \infty)$, and the initial random variable $r_{0}$ is distributed as $\pi$. 
A2 makes it possible to characterize the diffusion function by (2.3) and subsequently construct the nonparametric estimator based on that expression. Under A1, the candidate stationary distribution $\pi($.$) exists. Stationarity of the process follows from$ initializing it with the stationary distribution itself. We now make assumptions required to guarantee that the Central Limit Theorem to be used in the proofs of Theorems 2 and 3 holds.

ASSUMPTION A3: The observed data sequence $\left\{r_{i}, i=1, \ldots, n\right\}$ is a strictly

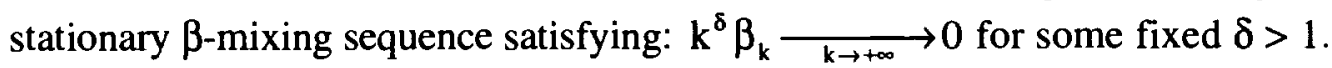

A3 restricts the amount of dependence allowed in the sequence. Without this assumption, the Central Limit Theorem can fail. As long as $\beta_{k} \underset{k \rightarrow+\infty}{\longrightarrow} 0$, the sequence is said to be absolutely regular. In order for the discretely observed process $\left\{r_{i}, i \geq 1\right\}$ to satisfy $A 3$, the stochastic process $\left\{r_{t}, t \geq 0\right\}$ has to verify a stronger continuous-time mixing condition (see Prakasa Rao (1987)). A condition on the drift and diffusion sufficient to insure that the discrete observations will satisfy A3 is the following:

ASSUMPTION A3':

$$
\lim _{r \rightarrow 0 \text { or } r \rightarrow \infty} \sigma(r) \pi(r)=0 \text { and } \lim _{r \rightarrow 0 \text { or } r \rightarrow \infty}\left|\sigma(r) /\left\{2 \mu(r, \theta)-\sigma(r) \sigma^{(1)}(r)\right\}\right|<\infty \text {. }
$$

Geometric ergodicity follows from A3'. Indeed under A3' Hansen and Scheinkman (1995) prove that the integral operator $T$ (such that the infinitesimal generator $A$ is the derivative of $\mathrm{T}$ ) is a strong contraction. This can be used here. It follows from the strong contraction property of $\mathrm{T}$ that there exists $\lambda, 0<\lambda<1$, such that for each measurable $\mathrm{f}$ and $g:\left|E\left[f\left(r_{i}\right) g\left(r_{i+k}\right)\right]\right| \leq\|f\|\|g\| \lambda^{k}$. Apply this to the indicator $f=1_{A}$ and $g=1_{B}$ to obtain the mixing bounds for the discrete data: $2 \alpha_{k} \leq \beta_{k} \leq \phi_{k} \leq \psi_{k} / 2 \leq \lambda^{k} / 2$ (where $\alpha, \beta, \phi$ and $\psi$ are the various classical mixing coefficients). This is clearly a stronger mixing property of the discrete data than what is strictly required by A3. Only A3 is necessary. The next assumption is the standard regularity condition imposed on a kernel function:

\section{ASSUMPTION A4:}

(i) The kernel $\mathrm{K}($.$) is an even function, continuously differentiable up to order \mathrm{r}$ on $R$ with $2 \leq r \leq s$, belongs to $L^{2}(R)$ and $\int_{-\infty}^{+\infty} K(x) d x=1$.

(ii) $\mathrm{K}(\cdot)$ is of order $\mathrm{r}: \int_{-\infty}^{+\infty} \mathrm{x}^{\mathrm{i}} \mathrm{K}(\mathrm{x}) \mathrm{dx}=0 \quad \mathrm{i}=1, \ldots, \mathrm{r}-1$, and: 


$$
\int_{-\infty}^{+\infty} x^{r} K(x) d x \neq 0, \quad \int_{-\infty}^{+\infty}|x|^{r}|K(x)| d x<\infty
$$

Note that in order to use effectively a kernel of order $r$ for bias reduction, the density $\pi$ must have at least that many derivatives. Hence the requirement in A1(i) that the drift and diffusion functions have $s \geq 2$ derivatives, and then the choice in A5 of $2 \leq r \leq s$. If a kernel of order $r=2$ is used, then only $s=2$ derivatives can be required in A1(i). More generally, when $s$ derivatives of $\pi$ exist, the optimal rate of convergence of the diffusion estimator is $n^{s /(2 s+1)}$. It is achieved by a kernel of order $r=s$ (even) with bandwidth set to $h_{n}=O\left(n^{-1 /(2 s+1)}\right)$. The optimal rate of convergence for $\pi$ and $\sigma^{2}$ can only be attained with a non-vanishing bias term (meaning that the asymptotic distribution of Theorem 2 would not be centered at 0 ). To avoid this undesirable feature, we require A5(i)-(ii):

ASSUMPTION A5 (Admissible Bandwidth Choices):

As $\mathrm{n} \rightarrow \infty, \mathrm{h}_{\mathrm{n}} \rightarrow 0$ and:

(i) To estimate $\pi: n^{1 / 2} h_{n}^{(2 r+1) / 2} \rightarrow 0$, and $n^{1 / 2} h_{n}^{1 / 2} \rightarrow \infty$.

(ii) To estimate $\sigma^{2}: n^{1 / 2} h_{n}^{(2 r+1) / 2} \rightarrow 0$, and $n^{1 / 2} h_{n}^{3 / 2} \rightarrow \infty$.

(iii) To estimate derivative prices $U: n^{1 / 2} h_{n}^{r} \rightarrow 0$.

A5 gives the admissible range of bandwidth choices depending upon the object to be estimated. The speed of decrease of the bandwidth $h_{n}$ to 0 as $n$ becomes large is restricted. Optimality within the respective admissible ranges is discussed later. In A5(i) the first condition insures that the bias in the normalized density estimator is asymptotically negligible. The second condition insures that the variance of the estimator which is of order $\mathrm{n}^{-1} \mathrm{~h}_{\mathrm{n}}^{-1}$ goes to zero. In A5(ii) the first condition serves the same purpose. Given the first, the second condition insures that the asymptotic distribution of the diffusion estimator is indeed given by the linear term in its Taylor expansion (i.e., the remainder term in the expansion is also asymptotically negligible). As a consequence of $n^{1 / 2} h_{n}^{2} \rightarrow \infty$, it follows that $n^{1 / 2} h_{n}^{1 / 2} \rightarrow \infty$ and therefore the variance of the estimator which is also of order $n^{-1} h_{n}^{-1}$ goes to zero. In A5(iii), the condition makes the bias negligible and is sufficient to make the remainder term in the Taylor expansion go to zero.

The empirical results for the density and diffusion estimators (Figure 2-3 and 4-5) are obtained with a Gaussian kernel $K(u)=\exp \left(-u^{2} / 2\right) / \sqrt{2 \pi}$ of order $r=2$, so we only need to assume the existence of $s=2$ derivatives of $\pi$. The optimal bandwidth choice (in the integrated mean squared error sense) within the range specified by either A5(i) or A5(ii) requires getting as close as possible to $\mathrm{n}^{-1 /(2 \mathrm{r}+1)}$. Choosing the bandwidth at this order 
would produce a bias term which does not vanish asymptotically. We therefore set the bandwidth as $h_{n}=c_{n} n^{-1 /(2 r+1)}=c_{n} n^{-1 / 5}$ where $c_{n}=c / \log (n)$ with the constant $c$ chosen to minimize the integrated mean square error of the diffusion estimator. The diffusion estimator can be calculated explicitly. For the actual sample size $n=5505$, the bandwidth was $h_{n}=1.603310^{-2}$.

The higher order kernel $K(u)=(3 / \sqrt{8 \pi})\left(1-u^{2} / 3\right) \exp \left(-u^{2} / 2\right)($ order $r=4)$ with optimal bandwidth $h_{n}=c_{n}^{\prime} n^{-1 /(1+2 r)}=c_{n}^{\prime} n^{-1 / 9}$, as well as exponential, sinusoidal, and Epanechnikov kernels produce similar results. The results are qualitatively robust to the choice of the kernel and small changes in the bandwidth parameter around the optimal value. Selection of the bandwidth by other various forms of cross-validation also produces similar estimates. The tail behavior of the kernel does not modify substantially the tail behavior of the diffusion estimator in the interval considered (the large values of the spot rate are clustered together in the sample so the tail behavior of the density is estimated fairly accurately up to $22 \%$ ).

The empirical results for the derivative prices (Tables 5-6 and Figure 7) are obtained with the same Gaussian kernel, but the optimal bandwidth choice in the integrated mean square error sense is now $h_{n}=\mathrm{cn}^{-1 / 3}$ where $\mathrm{c}$ is a scaling factor. The scaling factor is still set to minimize the integrated mean square error of the estimator. The precise choice of the bandwidth in this case does not modify the rate of convergence $n^{1 / 2}$ of derivative prices (it only affects higher order term in the integrated mean squared error). Hence as long as A5(iii) is satisfied it is substantially less important to be close to the optimal bandwidth than for pointwise estimation of $\sigma^{2}$. Finally note that the optimal bandwidth choice when estimating $\sigma^{2}$ pointwise is larger (less smoothing) than is admissible when estimating derivative prices. This is due to the respective sizes of the bias terms in the two cases. In particular the estimate of derivative prices would be biased if they were computed from plugging in the estimate of $\sigma^{2}$ computed at its pointwise bandwidth choice (A5'(i)). We summarize the actual bandwidth choice in:

ASSUMPTION A5' (Actual Bandwidth Choice):

(i) To estimate $\pi$ and $\sigma^{2}: \quad h_{n}=c_{n} n^{-1 /(2 r+1)}=c_{n} n^{-1 / 5}$ with $c_{n}=c / \log (n)$.

(ii) To estimate derivative prices $U: h_{n}=\mathrm{cn}^{-1 / 3}$.

(iii) In both cases, the scaling factor $c$ is set to minimize the asymptotic integrated mean squared error of the estimator. 
Monte-Carlo evidence shows that the asymptotic distribution given by Theorem 2 is a good approximation for the actual distribution of the diffusion estimator given the same sample size as the data set. The coverage probabilities are consistently above 0.85 when simulating data with various specifications (CIR, various power functions for the diffusion: $\left.\sigma^{2}(r)=r^{\gamma}\right)$ and computing the diffusion estimator.

\section{Appendix 2: Identification with a Generic Parametrization of the Drift}

Let $\mu(r, \theta)$ be a parametrization of the drift. The identification restriction states that there exists a unique parameter vector $\theta$ such that the true drift is $\mu(r, \theta)$. This Appendix shows how to modify (2.4) when $\mu$ is not necessarily linear. Let $\eta(r) \equiv \int_{0}^{r} \mu(u, \theta) \pi(u) d u$. Then the equation $\partial \vartheta(r, t) / \partial t=A \vartheta(r, t)$ with initial condition $\vartheta(r, 0)=r$ implies:

$$
\begin{aligned}
\pi(\mathrm{r}) \partial \vartheta / \partial \mathrm{t} & =\mu(\mathrm{r}, \theta) \pi(\mathrm{r}) \partial \vartheta / \partial \mathrm{x}+(1 / 2) \sigma^{2}(\mathrm{r}) \pi(\mathrm{r}) \partial^{2} \vartheta / \partial \mathrm{r}^{2} \\
& =\{\partial \eta / \partial \mathrm{r}\}\{\partial \vartheta / \partial \mathrm{r}\}+\eta(\mathrm{r})\left\{\partial^{2} \vartheta / \partial \mathrm{r}^{2}\right\}
\end{aligned}
$$

Therefore $\vartheta$ solves $\pi(r) \partial \vartheta / \partial t=\{\partial / \partial r\}[\eta(r) \partial \vartheta / \partial r]$. By Dynkin's formula again this partial differential equation has a unique solution given by $\vartheta(r, t)=E\left[r_{t} \mid r_{0}=r\right]$. Solving this equation yields $\vartheta$. Then $\theta$ can be estimated consistently by nonlinear least squares with this generalization of (2.4).

\section{Appendix 3: Regularity Conditions for the Pricing Equation}

The following assumptions are introduced to obtain nonparametric derivative prices and their asymptotic distribution. They are not needed to estimate the diffusion function itself.

ASSUMPTION A6: The drift, diffusion and density functions of the risk-neutral spot rate process, $\mathrm{dr}_{\mathrm{t}}=\left\{\mu\left(\mathrm{r}_{\mathrm{t}}, \theta\right)-\lambda\left(\mathrm{r}_{\mathrm{t}}\right) \sigma\left(\mathrm{r}_{\mathrm{t}}\right)\right\} \mathrm{dt}+\sigma\left(\mathrm{r}_{\mathrm{t}}\right) \mathrm{dW}$, satisfy the conditions in A1A2. 
ASSUMPTION A7: The derivative continuous payment rate $\mathrm{c}$ and final payoff $\mathrm{g}$ are right-continuous-left-limit functions and satisfy for $(r, t)$ in $(0, \infty) x(0, T]$ : $|c(r, t)| \leq N \exp \left(v r^{2}\right)$ and $|g(r)| \leq N \exp \left(v r^{2}\right)$ for some $v, N>0$.

ASSUMPTION A8: The derivative price $U(r, t)$ satisfies the exponential boundedness condition: $\int_{0}^{\mathrm{T}} \int_{0}^{+\infty} \mathrm{e}^{-\mathrm{kr}^{2}}|\mathrm{U}(\mathrm{r}, \mathrm{t})| \mathrm{dr} \mathrm{dt}<\infty$ for some $\mathrm{k}>0$.

The solution to (4.1) is only unique in the class of functions satisfying A8. The bound is sharp as the solution fails to be unique in general if A8 is replaced by $\int_{0}^{\mathrm{T}} \int_{0}^{+\infty} \mathrm{e}^{-\mathrm{kr}^{2+\varepsilon}}|\mathrm{U}(\mathrm{r}, \mathrm{t})| \mathrm{dr} \mathrm{dt} \quad<\infty$ for some $\varepsilon>0$ (see Friedman (1975)).

ASSUMPTION A9: The market price of interest rate risk depends on a parameter $\lambda$ and is estimated by minimizing the sum of squared deviations between a fixed set of derivative prices and the corresponding nonparametric prices.

\section{Appendix 4: Solving the Pricing Partial Differential Equation}

The partial differential equation (4.1) with (4.3) and then (4.4) will be solved numerically using finite-differencing with a combination of an implicit scheme for the advective term $\partial U / \partial r$ and the diffusion term $\partial^{2} U / \partial r^{2}$. Since the numerical solution is guaranteed to converge only on a compact set, we make the change of variable $\mathrm{s} \equiv \mathrm{cr} /(1+\mathrm{cr})$ where the constant $\mathrm{c}$ is large enough to over-represent interest rate levels between 0 and $20 \%$. This increases the accuracy of the numerical procedure. Let $\mathrm{V}(\mathrm{s}, \mathrm{t}) \equiv \mathrm{U}(\mathrm{r}, \mathrm{t})$, so we can replace in $(4.1): \partial \mathrm{U} / \partial \mathrm{t}=(\partial \mathrm{V} / \partial \mathrm{t}), \partial \mathrm{U} / \partial \mathrm{r}=(\partial \mathrm{V} / \partial \mathrm{s})(\mathrm{ds} / \mathrm{dr})$ and $\partial^{2} \mathrm{U} / \partial \mathrm{r}^{2}=\left(\partial^{2} \mathrm{~V} / \partial \mathrm{s}^{2}\right)(\mathrm{ds} / \mathrm{dr})^{2}+(\partial \mathrm{V} / \partial \mathrm{s})\left(\mathrm{d}^{2} \mathrm{~s} / \mathrm{dr}^{2}\right)$. The discretized counterpart to (4.1) is:

$$
\begin{aligned}
& \frac{v_{j}^{m}-v_{j}^{m-1}}{\Delta t}+p_{j}\left(\frac{\left(v_{j+1}^{m}-2 v_{j}^{m}+v_{j-1}^{m}\right)+\left(v_{j+1}^{m-1}-2 v_{j}^{m-1}+v_{j-1}^{m-1}\right)}{2(\Delta s)^{2}}\right) \\
& +q_{j}\left(\frac{\left(v_{j+1}^{m}-v_{j-1}^{m}\right)+\left(v_{j+1}^{m-1}-v_{j-1}^{m-1}\right)}{4 \Delta s}\right)-r\left(s_{j}\right) \frac{v_{j}^{m}+v_{j}^{m-1}}{2 \Delta s}+c\left(r\left(s_{j}\right), t^{m}\right)=0
\end{aligned}
$$


where $\mathrm{v}_{j}^{\mathrm{m}} \equiv \mathrm{V}\left(\mathrm{s}_{\mathrm{j}}, \mathrm{t}^{\mathrm{m}}\right)$, and $\mathrm{t}^{\mathrm{m}}$ and $\mathrm{s}_{\mathrm{j}}$ are the discretized values of the time and spot interest rate transformed variable, $j=2, \ldots, J-1$ and $m=1, \ldots, M . r\left(s_{j}\right)$ is the inverted change of variable. The coefficients $p$ and $q$ can easily be obtained from (4.1) and the differentials above. For $\mathrm{j}=1$, corresponding to 0 , the diffusion function is zero and thus the equation becomes: $\frac{v_{1}^{m}-v_{1}^{m-1}}{\Delta t}+q_{1}\left(\frac{\left(v_{2}^{m}-v_{1}^{m}\right)+\left(v_{2}^{m-1}-v_{1}^{m-1}\right)}{4 \Delta s}\right)+d\left(0, t^{m}\right)=0$. For $j=J, v_{j}^{m}$ is obtained explicitly from $v_{j}^{m-1}, v_{J-1}^{m-1}$ and $v_{J-2}^{m-1}$.

Obviously, the equation could be solved more easily with a fully explicit representation. However, explicit finite-differencing of parabolic equations is known to yield estimates that do not necessarily converge to the true solution of the equation (see Ames (1977) for a von Neumann stability analysis). We have adopted the method recommended by Ames (1977) consisting of an implicit discretization of the second order partial derivative and an explicit discretization of the first order derivatives. This method is known as the Crank-Nicolson scheme. Convergence to the solution as $\Delta x \rightarrow 0$ and $\Delta t \rightarrow 0$ is guaranteed. For given $\left\{u_{j}^{m} / j=1, \ldots, J\right\},(7.1)$ gives $\left\{u_{j}^{m-1} / j=1, \ldots, J\right\}$ as the solution of an easily solvable triangular system. The final set $\left\{u_{j}^{M} / j=1, \ldots, J\right\}$ is derived directly from the final condition (4.3) for bond pricing, and later (4.4) for bond option pricing. Then the equation is used recursively backwards to derive successively the sets $\left\{u_{j}^{m} / j=1, \ldots, J\right\}$ for $\mathrm{m}=\mathrm{M}-1, \mathrm{M}-2$, etc, up to $\mathrm{m}=1$.

When actually pricing bonds and bond options under the CIR model and comparing the numerical solutions to the closed-form formulas, this procedure was found to be very accurate, and the approximation error to shrink as much as wanted when decreasing the step sizes.

\section{Appendix 5: Proofs}

PROOF OF THEOREM 2: The proof uses the functional delta results of AïtSahalia (1992). The diffusion function (2.3) is viewed as a functional of the joint cumulative distribution function $F$ of observations sampled $\Delta=1$ day apart, $\left(r_{t}, r_{t+\Delta}\right)$. Compute a first-order expansion of the diffusion away from F. From (2.3):

$$
\sigma^{2}[\mathrm{~F}+\mathrm{H}](\mathrm{x})=\frac{2}{\pi[\mathrm{F}+\mathrm{H}](\mathrm{x})} \int_{0}^{\mathrm{x}} \mu(\mathrm{u}, \theta[\mathrm{F}+\mathrm{H}]) \pi[\mathrm{F}+\mathrm{H}](\mathrm{u}) \mathrm{du}
$$


The drift parameters are estimated at the parametric speed, i.e., $\theta($.$) is a regular$ functional. That is, there exists a linear functional $\theta^{(1)}(\cdot, \cdot) \in \mathrm{C}^{-1}$ such that $\theta[F+H]=\theta[F]+\int_{0}^{+\infty} \int_{0}^{+\infty} \theta^{(1)}(u, v) h(u, v) d u d v+O\left(\|H\|_{L(\infty, 1)}^{2}\right) . C^{-1}$ is the space of cadlag functions. The norm $\mathrm{L}(\infty, 1)$ is the sum of the supremum norms for $\mathrm{H}$ and its derivative $h$. The fact that $\theta($.) is evaluated at the empirical distribution instead of a kernel plug-in does not change the asymptotic distribution.

Let $\delta_{(x)}$ denote a Dirac mass centered at $x$. The expansion for the marginal density is: $\pi[F+H](x)=\pi[F](x)+\int_{0}^{+\infty} \int_{0}^{+\infty} \delta_{(x)}(u) h(u, v) d u d v+O\left(\|H\|_{L(\infty, 1)}^{2}\right)$. Collecting terms:

$$
\sigma^{2}[F+H](x)=\sigma^{2}[F](x)+\int_{-\infty-\infty}^{+\infty+\infty}\left\{\frac{1}{\pi(x)}\left(-\sigma^{2}(x) \delta_{(x)}(u, v)+B[F](x)\right)\right\} h(u, v) d u d v+O\left(\|H\|_{L(\infty, 1)}^{2}\right)
$$

$\mathrm{B}(\mathrm{F})(\cdot)$ is a functional in $\mathrm{C}^{-1}$ which does not influence the asymptotic distribution, and therefore need not be identified. It follows that $\sigma^{2}[].(x)$ is $L(\infty, 1)$-differentiable with respect to $F$. That is, there exists a distribution $\varphi(F)(.,$.$) such that the functional \sigma^{2}($. satisfies: $\quad \sigma^{2}[\mathrm{~F}+\mathrm{H}](\mathrm{x})=\sigma^{2}[\mathrm{~F}](\mathrm{x})+\int_{0}^{+\infty} \int_{0}^{+\infty} \varphi[\mathrm{F}](\mathrm{u}, \mathrm{v}) \mathrm{h}(\mathrm{u}, \mathrm{v}) \mathrm{dudv}+\mathrm{O}\left(\|\mathrm{H}\|_{\mathrm{L}(\infty, 1)}^{2}\right) . \quad$ The derivative of the functional $\sigma^{2}($.$) is \varphi[F](u, v)=\left\{\frac{-\sigma^{2}[F](x)}{\pi[F](x)}\right\} \delta_{(x)}(u, v)+B(x) \in C^{-2} \backslash C^{-1}$ where $\mathrm{C}^{-2}$ is the space of Dirac delta functions. Its asymptotic distribution follows directly from the functional delta method.

PROOF OF LEMMA 1: $\quad$ Consider the compact set $K_{\varepsilon}=(\varepsilon, 1 / \varepsilon)$ for a fixed $\varepsilon>0$, and fix $x$ in $K_{\varepsilon}$. Consider the equation $L_{\varepsilon} \Gamma=0$, where $L_{\varepsilon} U \equiv L U+\varepsilon\left(\partial^{2} U / \partial r^{2}\right)$. It is necessary to perturbate $L$ slightly because $\sigma^{2}(r)>0$ for all $r>0$ but $\sigma^{2}(0)=0$ thus $L$ is parabolic on $(0, \infty) \times(0, T]$ instead of the entire domain $(0, \infty) \times(0, T]$. Under $A 6, L_{\varepsilon}$ is uniformly parabolic on $\mathrm{K}_{\varepsilon} \mathrm{x}(0, \mathrm{~T}]$ and its coefficient functions satisfy the hypotheses of Friedman (1964), Section I.4. We can therefore construct the fundamental solution $\Gamma_{\varepsilon}$ corresponding to the operator $\mathrm{L}_{\varepsilon}$ by using the parametrix method. Alternatively, $\Gamma_{\varepsilon}$ could be obtained as the limit of the Green functions associated with $L_{\varepsilon}$ on $K_{\varepsilon} x(0, T]$ as in Friedman (1976), Lemma 15.1.1; see also Freidlin (1985).

Form the parametrix solution for any $(r, t)$ in $K_{\varepsilon} \times(0, T]$ : $\Gamma_{\varepsilon}(\mathrm{r}, \mathrm{t} ; \mathrm{x})=\mathrm{Z}(\mathrm{r}, \mathrm{t} ; \mathrm{x})+\int_{0}^{\mathrm{t}} \int_{\varepsilon}^{1 / \varepsilon} \mathrm{Z}(\mathrm{r}, \mathrm{t}-\mathrm{s} ; \mathrm{y}) \Phi_{\varepsilon}(\mathrm{y}, \mathrm{s} ; \mathrm{x}) \mathrm{dyds}$ where we have introduced $\mathrm{Z}(\mathrm{r}, \mathrm{t} ; \mathrm{x}) \equiv(1 / \sqrt{2 \pi \mathrm{t}} \sigma(\mathrm{x})) \exp \left\{-(\mathrm{r}-\mathrm{x})^{2} / 2 \mathrm{t} \sigma^{2}(\mathrm{x})\right\}$ and $\Phi_{\varepsilon}(\mathrm{y}, \mathrm{s} ; \mathrm{x}) \equiv \sum_{\mathrm{p}=1}^{\infty}\left(\mathrm{L}_{\varepsilon} \mathrm{Z}\right)_{\mathrm{p}}(\mathrm{y}, \mathrm{s} ; \mathrm{x})$ 
The differential operator $\left(\mathrm{L}_{\varepsilon} \cdot\right)_{\mathrm{p}}$ is defined recursively by: $\left(\mathrm{L}_{\varepsilon} \mathrm{Z}\right)_{1}=\mathrm{L}_{\varepsilon} \mathrm{Z}$ and $\left(\mathrm{L}_{\varepsilon} \mathrm{Z}\right)_{\mathrm{p}+1}(\mathrm{y}, \mathrm{s} ; \mathrm{x})=\int_{0}^{\mathrm{s}} \int_{\varepsilon}^{1 / \varepsilon}\left[\mathrm{L}_{\varepsilon} \mathrm{Z}(\mathrm{y}, \mathrm{s}-\tau ; \mathrm{z})\right]\left[\left(\mathrm{L}_{\varepsilon} \mathrm{Z}\right)_{\mathrm{p}}(\mathrm{z}, \tau ; \mathrm{x})\right] \mathrm{dzd} \tau$. By Theorem I.8 in Friedman (1964) the infinite series in $\Phi_{\varepsilon}$ converges and the parametrix solution is well-defined.

Next it is clear that the parametrix expression is a fundamental solution of (4.1) on $\mathrm{K}_{\varepsilon} \mathrm{x}(0, \mathrm{~T}]$. Write:

$$
\begin{aligned}
\mathrm{L}_{\varepsilon} \Gamma_{\varepsilon}(\mathrm{r}, \mathrm{t} ; \mathrm{x}) & =\mathrm{L}_{\varepsilon} \mathrm{Z}(\mathrm{r}, \mathrm{t} ; \mathrm{x})+\mathrm{L}_{\varepsilon}\left\{\int_{0}^{\mathrm{t}} \int_{\varepsilon}^{1 / \varepsilon} \mathrm{Z}(\mathrm{r}, \mathrm{t}-\mathrm{s} ; \mathrm{y}) \Phi_{\varepsilon}(\mathrm{y}, \mathrm{s} ; \mathrm{x}) \mathrm{dyds}\right\} \\
& =\mathrm{L}_{\varepsilon} \mathrm{Z}(\mathrm{r}, \mathrm{t} ; \mathrm{x})-\Phi_{\varepsilon}(\mathrm{r}, \mathrm{t} ; \mathrm{x})+\int_{0}^{\mathrm{t}} \int_{\varepsilon}^{1 / \varepsilon} \mathrm{L}_{\varepsilon} \mathrm{Z}(\mathrm{r}, \mathrm{t}-\mathrm{s} ; \mathrm{y}) \Phi_{\varepsilon}(\mathrm{y}, \mathrm{s} ; \mathrm{x}) \mathrm{dyds}
\end{aligned}
$$

Thus for $\Gamma_{\varepsilon}$ to be a fundamental solution $\Phi_{\varepsilon}$ must satisfy the Volterra integral equation: $\Phi_{\varepsilon}(r, t ; x)=L_{\varepsilon} Z(r, t ; x)+\int_{0}^{t} \int_{\varepsilon}^{1 / \varepsilon} L_{\varepsilon} Z(r, t-s ; y) \Phi_{\varepsilon}(y, s ; x) d y d s$. By construction the Volterra equation is satisfied by $\sum_{p=1}^{\infty}\left(L_{\varepsilon} Z\right)_{p}(r, t ; x)$.

Then as in Theorem 15.1.2 in Friedman (1976) we obtain that as $\varepsilon$ goes to zero $\Gamma_{\varepsilon}(r, t ; x)$ converges to a limit $\Gamma(r, t ; x)$, together with the first two r-derivatives, $x$ derivatives and the first $t$-derivative uniformly for all $r$ and $x$ in $(0, \infty)$ and $t$ in $(0, T]$. Furthermore the limit $\Gamma(\mathrm{r}, \mathrm{t} ; \mathrm{x})$ satisfies $(4.1)$ on $(0, \infty) \mathrm{x}(0, \mathrm{~T}]$ as in Corollary 15.1.3 in Friedman (1976). The fundamental solution has the form $\Gamma(\mathrm{r}, \mathrm{t} ; \mathrm{x})=\gamma\left(\mathrm{r}, \mathrm{t}, \mathrm{x}, \sigma^{2}(\mathrm{x}), \mu(\mathrm{x})\right)$ with $\gamma$ continuously differentiable in each of its arguments (see the parametrix form). The only difference here with the results in Chapter 15 in Friedman (1976) is that the parabolic operator $\mathrm{L}$ contains the term (-rU). The proof is otherwise identical. Finally the fundamental solution is unique in the class of non-explosive solutions (assumption A8). By Theorem I.16 in Friedman (1964), for each $\varepsilon>0 \Gamma_{\varepsilon}$ is unique on $K_{\varepsilon} x(0, T]$. Any two solutions on $(0, \infty) \times(0, T]$ would have to coincide on $K_{\varepsilon} x(0, T]$.

PROOF OF THEOREM 3: We first consider the case where the market price of risk is known, or without lack of generality $\lambda \equiv 0$. Then the coefficients of the differential operator $L$ are functionals of the joint cdf $F$ of the data $\left(r_{t}, r_{t+\Delta}\right)$ so the differential operator is $L[F]$. Then consider the equations followed by the derivative prices associated to $F$ and $\mathrm{F}+\mathrm{H}$ respectively: $\mathrm{L}[\mathrm{F}] \mathrm{U}[\mathrm{F}](\mathrm{r}, \mathrm{t})=-\mathrm{d}(\mathrm{r}, \mathrm{t})$ with initial condition $\mathrm{U}[\mathrm{F}](\mathrm{r}, 0)=\mathrm{g}[\mathrm{F}](\mathrm{r})$, and $L[F+H] U[F+H](r, t)=-d(r, t)$ with initial condition: $U[F+H](r, 0)=g[F+H](r)$. The final payoff $\mathrm{g}($.) depends on $\mathrm{F}$ because it could be the price of a derivative previously computed: for example, the price of the underlying bond when valuing a bond option. 
Lemma 1 characterizes the fundamental solution $\Gamma$ in the form $\Gamma(\mathrm{r}, \mathrm{t} ; \mathrm{x})=\gamma\left(\mathrm{r}, \mathrm{t}, \mathrm{x}, \sigma^{2}(\mathrm{x}), \mu(\mathrm{x})\right)$ with $\gamma$ continuously differentiable in each of its arguments. From this characterization, and the (linear in $\mathrm{H}$ ) expansions of the drift and diffusion functionals in Theorem 2, it follows that $\Delta U[F, H] \equiv U[F+H]-U[F]$ admits the expansion $(L(\infty, 0)$ is the standard supremum norm for $H)$ :

$$
\begin{aligned}
\Delta \mathrm{U}[\mathrm{F}, \mathrm{H}](\mathrm{r}, \mathrm{t}) & =\int_{0}^{+\infty} \Gamma[\mathrm{F}+\mathrm{H}](\mathrm{r}, 0 ; \mathrm{x}) \mathrm{g}[\mathrm{F}+\mathrm{H}](\mathrm{x}) \mathrm{dx}-\int_{0}^{+\infty} \Gamma[\mathrm{F}](\mathrm{r}, 0 ; \mathrm{x}) \mathrm{g}[\mathrm{F}](\mathrm{x}) \mathrm{dx} \\
& +\int_{0}^{\mathrm{t}} \int_{0}^{+\infty}\{\Gamma[\mathrm{F}+\mathrm{H}]-\Gamma[\mathrm{F}]\}(\mathrm{r}, \tau ; \mathrm{x}) \mathrm{d}(\mathrm{x}, \tau) \mathrm{dxd} \tau \\
& =\int_{0}^{+\infty} \int_{0}^{+\infty} \mathrm{u}[\mathrm{F}](\mathrm{r}, \mathrm{t}, \mathrm{x}, \mathrm{y}) \mathrm{h}(\mathrm{x}, \mathrm{y}) \mathrm{dxdy}+\mathrm{O}\left(\|\mathrm{H}\|_{\mathrm{L}(\infty, 0)}^{2}\right)
\end{aligned}
$$

Suppose that for the derivative under consideration the final payoff $g($.$) is$ independent of $F$ (e.g., a bond for which $g=1$, or an interest rate option: $g=\max (0, r-k)$ ). Then the functional $F \mapsto U[F](r, t)$ for fixed $(r, t)$ in $(0, \infty) x(0, T]$ is $L(\infty, 1)$-differentiable with respect to $\mathrm{F}$ with a continuous functional derivative. Its functional derivative $\mathrm{u}($.$) is$ continuous as can be seen from collecting terms from the linearizations of $\mu($.$) and \sigma^{2}($. (which were computed in Theorem 2) and the partial derivatives of $\gamma\left(r, t, x, \sigma^{2}(x), \mu(x)\right)$ with respect to $\sigma^{2}$ and $\mu$. If now the derivative's payoff $g$ depends on $F$, typically through the previously computed price of a more primitive derivative, then the expansion of $g($. also matters. An example of this situation is a bond call option where $g(F)$ is the maximum of zero and the price of the underlying bond (dependent on F) net of the strike price. By what precedes, the bond price admits a first-order expansion in $\mathrm{F}$ with derivative in $\mathrm{C}^{-1}$ (since its payoff is independent of F). Under A7 the final payoff for the derivative also satisfies this requirement. The functional derivative $u($.$) then has additional terms which$ reflect this additional dependence on $\mathrm{F}$.

We next show that the introduction of the market price of risk does not modify the rate of convergence (only the asymptotic variance). This holds provided that $\lambda$ is estimated at a speed ${ }^{9}$ that is at least as fast as that of $\sigma^{2}$, in particular when the estimator of $\lambda$ converges at speed root-n. This will hold as a further special case when the market price of risk is estimated by matching the prices a cross-section of derivatives $\left(\left\{\mathbf{U}_{i}\right\}, \mathrm{i}=1, \ldots, \mathrm{J}\right)$. Indeed under A9 set $\hat{\lambda} \equiv \arg \min _{\lambda \in \mathrm{L}} \sum_{\mathrm{j}=1}^{\mathrm{J}}\left\{\hat{\mathrm{U}}_{\lambda}\left(\mathrm{r}, \mathrm{t}_{\mathrm{i}}\right)-\mathrm{U}_{\mathrm{i}}\right\}^{2}$ by minimizing the squared

9 If however $\lambda$ were estimated at a slower speed than that of $\sigma^{2}$ (e.g., its derivative were in $C^{-3} \backslash C^{-2}$, the space of first derivatives of Dirac masses) then $\mathrm{U}[. .$.$] would no longer converge at speed root-n.$ 
deviations between the derivative prices $\left(\left\{U_{i}\right\}\right)$ and those predicted by the model $\left(\hat{U}_{\lambda}\left(r, t_{i}\right)\right.$ evaluated at $\lambda$ ).

Note that under A9 we assume that we are matching a fixed set of derivative prices and therefore do not have to account for the variability of prices being matched ${ }^{10}$. Recall that the solution $U_{\lambda}$ has $\mu$ replaced by $\mu-\lambda \sigma$. Furthermore the characterization of the fundamental solution has $\Gamma(\mathrm{r}, \mathrm{t} ; \mathrm{x})=\gamma\left(\mathrm{r}, \mathrm{t}, \mathrm{x}, \sigma^{2}(\mathrm{x}), \mu(\mathrm{x})\right)$ with $\gamma$ continuously differentiable in each of its arguments. Therefore $\hat{U}_{\lambda}\left(r, t_{i}\right)$ is continuously differentiable in $\lambda$ and $\lambda($.$) is$ $\mathrm{L}(\infty, 1)$-differentiable as a functional of $\mathrm{F}$ with derivative in $\mathrm{C}^{-1}$ by the implicit functional theorem: view $\lambda$ as the implicit solution of the first order condition: $\sum_{j=1}^{J}\left\{\partial \hat{U}_{\lambda}\left(r, t_{i}\right) / \partial \lambda\right\}\left\{\hat{U}_{\lambda}\left(r, t_{i}\right)-U_{i}\right\}=0$. As a consequence, this particular $\hat{\lambda}$ converges at speed root-n and $\hat{U}$ also converges at root-n. Collect all the linear terms in the first-order expansion of $U$ and let $u_{(r, t)}$ be the derivative (in $\mathrm{C}^{-1}$ by what precedes) of $U$. A complete expression for $u_{(r, t)}$ can be computed explicitly but is not needed since the asymptotic variance of the prices will be obtained by the bootstrap.

10 If we were matching a random set of derivative prices, such as the term structure on a particular day, then derivatives prices would depend not only on $F$ but also on $G$, the joint cdf of the prices being matched. For example $G$ would be the joint cdf of discount bond prices at the fixed maturities $t_{1}, \ldots, t_{\mathrm{J}}$. A formal proof in that case goes beyond the scope of this paper. 


\section{REFERENCES}

AIT-SAHALIA, Y. (1992): "The Delta and Bootstrap Methods for Nonparametric Kernel Functionals," MIT Mimeo, January.

---- (1994): "Testing Continuous-Time Models of the Spot Interest Rate," forthcoming in the Review of Financial Studies.

AMES, W.F. (1977): Numerical Methods for Partial Differential Equations, Academic Press, New-York.

BANON, G. (1978): "Nonparametric Identification for Diffusion Processes," S.I.A.M. Journal of Control and Optimization, 16, 380-395.

BASAWA, I.V. AND B.L.S. PRAKASA-RAO (1980): Statistical Inference for Stochastic Processes, New York: Academic Press.

BLACK, F. AND M. SCHOLES (1973): "The Pricing of Options and Corporate Liabilities," Journal of Political Economy, 3, 133-155.

BRENNAN, M.J. AND E.S. SCHWARTZ (1979), "A Continuous-Time Approach to the Pricing of Bonds," Journal of Banking and Finance, Vol. 3, 133-155.

BROWN, S.J. AND P.H. DYBVIG (1986): "The Empirical Implications of the Cox, Ingersoll, Ross Theory of the Term Structure of Interest Rates," Journal of Finance, 41, 3, 617-630.

CHAMBERLAIN, G. (1987): "Asymptotic Efficiency in Estimation with Conditional Moment Restrictions," Journal of Econometrics, 34, 305-334.

CHAN, K.C., G.A. KAROLYI, F.A. LONGSTAFF AND A.B. SANDERS (1992): "An Empirical Comparison of Alternative Models of the Short-Term Interest Rate," Journal of Finance, 47, 3, 1209-1227.

CONSTANTINIDES, G.M. (1992): "A Theory of the Nominal Term Structure of Interest Rates," Review of Financial Studies, 5, 4, 531-552.

COURTADON, G. (1982): "The Pricing of Options on Default-Free Bonds," Journal of Financial and Quantitative Analysis, 17, 1, 75-100.

COX, J.C. (1975): "Notes on Option Pricing I: Constant Elasticity of Diffusions," Stanford University Mimeo, September 1975.

COX, J.C., J.E. INGERSOLL AND S.A. ROSS (1980): "An Analysis of Variable Rate Loan Contracts," Journal of Finance, 35, 2, 389-403.

(1985a): "An Intertemporal General Equilibrium

Model of Asset Prices," Econometrica, 53, 2, 363-384.

Interest Rates," Econometrica, 53, 2, 385-407.

DOTHAN, L.U. (1978): "On the Term Structure of Interest Rates," Journal of Financial Economics, 6, 59-69.

DUFFIE, D. AND R. KAN (1993): "A Yield Factor Model of Interest Rates," Stanford University Mimeo.

DUFFIE, D. AND K. SINGLETON (1993): "Simulated Moments Estimation of Markov Models of Asset Prices," Econometrica, 61, 4, 929-952.

FELLER, W. (1951): "Two Singular Diffusion Problems," Annals of Mathematics, 54, 2, 173-182.

FLORENS-ZMIROU, D. (1993): "On Estimating the Diffusion Coefficient from Discrete Observations," Journal of Applied Probability, 30, 790-804.

FREIDLIN, M. (1985): Functional Integration and Partial Differential Equations, Annals of Mathematics Studies, Number 109, Princeton, N.J.: Princeton University Press. FRIEDMAN, A. (1964): Partial Differential Equations of Parabolic Type, Englewood Cliff, N.J.: Prentice-Hall. 
(1975): Stochastic Differential Equations and Applications, Vol. 1, New York: Academic Press.

- (1976): Stochastic Differential Equations and Applications, Vol. 2, New York: Academic Press.

GIBBONS, M.R. AND K. RAMASWAMY (1993): "A Test of the Cox, Ingersoll and Ross Model of the Term Structure," The Review of Financial Studies, 6, 3, 619-658.

GOURIEROUX, C., A. MONFORT AND E. RENAULT (1993): "Indirect Inference," Journal of Applied Econometrics, Vol. 8, S85-S118.

HANSEN, L.P. (1982): "Large Sample Properties of Generalized Method of Moments Estimators," Econometrica, 50, 1029-1054.

HANSEN, L.P. AND J.A. SCHEINKMAN (1995): "Back to the Future: Generating Moment Implications for Continuous Time Markov Processes," forthcoming in Econometrica.

HARVEY, A.C. (1993): Time Series Models, Second Edition, Cambridge, MA: The MIT Press.

HASMINSKII, R.Z. (1980): Stochastic Stability of Differential Equations, Alphen aan den Rijn, The Netherlands: Sijthoff and Noordhoff Publishers.

HULL, J. AND A. WHITE (1990): "Pricing Interest-Rate-Derivative Securities," The Review of Financial Studies, 3, 4, 573-592.

(1990): "Valuing Derivative Securities Using the Explicit Finite Difference Method," The Journal of Financial and Quantitative Analysis, 25, 1, 87-100.

JAMSHIDIAN, F. (1989): "An Exact Bond Option Formula," Journal of Finance, 44, 1, 205-209.

KARATZAS, I. AND S.E. SHREVE (1991): Brownian Motion and Stochastic Calculus, Second Edition, New York: Springer-Verlag.

KARLIN, S. AND H.M. TAYLOR (1981): A Second Course in Stochastic Processes, New York: Academic Press.

KÜNSCH, H.R (1989): "The Jacknife and the Bootstrap for General Stationary Observations," Annals of Statistics, 17, 3, 1217-1241.

LIU, R.Y. AND K. SINGH (1992): "Moving Blocks Jacknife and Bootstrap Capture Weak Dependence," in LePage R. and Billard, Y., eds, Exploring the Limits of Bootstrap, New York: Wiley.

LO, A.W. (1988): "Maximum Likelihood Estimation of Generalized Itô Processes with Discretely Sampled Data," Econometric Theory, 4, 231-247.

MARSH, T.A. AND E.R. ROSENFELD (1983): "Stochastic Processes for Interest Rates and Equilibrium Bond Prices," Journal of Finance, 38, 2, 635-646.

MERTON, R.C. (1990): Continuous-Time Finance, Cambridge, MA: Blackwell.

(1980): "On Estimating the Expected Return on the Market: An

Exploratory Investigation," Journal of Financial Economics, 8, 323-361.

(1973): "Theory of Rational Option Pricing," Bell Journal of Economics

and Management Science, 4, 141-183.

NELSON, D.B. (1990): "ARCH Models as Diffusion Approximations," Journal of Econometrics, 45, 1/2, 7-38.

ØKSENDAL, B. (1992): Stochastic Differential Equations, Third Edition, New York: Springer-Verlag.

PEARSON, N.D. AND T.-S. SUN (1994): "Exploiting the Conditional Density in Estimating the Term Structure: An Application to the Cox, Ingersoll, and Ross Model," Journal of Finance, 49, 4, 1279-1304.

PHILLIPS, P.C.B. (1987): "Time Series Regression with a Unit Root," Econometrica, 55, 2, 277-310.

PRAKASA-RAO, B.L.S. (1987): "On Mixing for Flows of $\sigma$-Algebras," Technical Report No. 95, University of California, Davis. 
ROBINSON, P.M. (1983): "Nonparametric Estimators for Time Series," Journal of Time Series Analysis, 4, 3, 185-207.

(1987): "Asymptotically Efficient Estimation in the Presence of Heteroskedasticity of Unknown Form," Econometrica, 55, 4, 875-891.

SAID, E.S. AND D.A. DICKEY (1984): "Testing for Unit Roots in AutoregressiveMoving Average Models of Unknown Order," Biometrika, 71, 3, 599-607.

SCOTT, D.W. (1992): Multivariate Density Estimation: Theory, Practice and Visualization, New York: Wiley.

SILVERMAN, B.W. (1986): Density Estimation for Statistical and Data Analysis, London: Chapman-Hall.

VASICEK, O. (1977): "An Equilibrium Characterization of the Term Structure," Journal of Financial Economics, 5, 177-188.

WONG, E. (1964): "The Construction of a Class of Stationary Markov Processes," in Stochastic Processes in Mathematical Physics and Engineering, Proceedings of Symposia in Applied Mathematics, Vol. XVI. 
Table 1: Alternative Specifications of the Spot Interest Rate Process

$$
d r_{t}=\mu\left(r_{t}\right) d t+\sigma\left(r_{t}\right) d W_{t}
$$

\begin{tabular}{|c|c|c|c|}
\hline $\begin{array}{c}\text { Drift } \\
\text { Function } \\
\mu(\mathbf{r})\end{array}$ & $\begin{array}{c}\text { Diffusion } \\
\text { Function } \\
\sigma(\mathbf{r})\end{array}$ & Stationary & Reference \\
\hline$\beta(\alpha-\mathrm{r})$ & $\sigma$ & Yes & Vasicek (1977) \\
\hline$\beta(\alpha-\mathrm{r})$ & $\sigma \mathrm{r}^{1 / 2}$ & Yes & $\begin{array}{c}\text { Cox-Ingersoll-Ross (1985b) } \\
\text { Brown-Dybvig (1986) } \\
\text { Gibbons-Ramaswamy (1993) }\end{array}$ \\
\hline$\beta(\alpha-\mathrm{r})$ & $\sigma \mathrm{r}$ & Yes & Courtadon (1982) \\
\hline$\beta(\alpha-\mathrm{r})$ & $\sigma \mathrm{r}^{\lambda}$ & Yes & Chan et al. (1992) \\
\hline$\beta(\alpha-\mathrm{r})$ & $\sqrt{\sigma+\gamma \mathrm{r}}$ & Yes & Duffie-Kan (1993) \\
\hline$\beta \mathrm{r}(\alpha-\mathrm{Ln}(\mathrm{r}))$ & $\sigma \mathrm{r}$ & Yes & Brennan-Schwartz (1979) [one-factor] \\
\hline$\alpha \mathrm{r}^{-(1-\delta)}+\beta \mathrm{r}$ & $\sigma \mathrm{r}^{\delta / 2}$ & Yes & Marsh-Rosenfeld (1983) \\
\hline$\alpha+\beta \mathrm{r}+\gamma \mathrm{r}^{2}$ & $\sigma+\gamma \mathrm{r}$ & Yes & Constantinides (1992) \\
\hline$\beta$ & $\sigma$ & No & Merton (1973) \\
\hline 0 & $\sigma \mathrm{r}$ & No & Dothan (1978) \\
\hline 0 & $\sigma \mathrm{r}^{3 / 2}$ & No & Cox (1975) \\
\hline & & & Cox-Ingersoll-Ross (1980) \\
\hline
\end{tabular}


Table 2: Descriptive Statistics

\begin{tabular}{|c|c|c|}
\hline Source & \multicolumn{2}{|c|}{$\begin{array}{l}\text { Bank of America 7-Day Eurodollar } \\
\text { (Deposit Rate Midpoint Bid-Ask) }\end{array}$} \\
\hline Frequency & \multicolumn{2}{|c|}{ Daily } \\
\hline Sample Period & \multicolumn{2}{|c|}{ 06/01/1973-2/25/1995 } \\
\hline Sample Size & \multicolumn{2}{|c|}{5505 observations } \\
\hline \multirow[t]{2}{*}{ Type } & \multicolumn{2}{|c|}{$\begin{array}{l}\text { Continuously-Compounded Yield-to-Maturity } \\
\text { (annualized rate) }\end{array}$} \\
\hline & Spot Interest Rate & $\begin{array}{l}\text { First Difference of } \\
\text { Spot Interest Rate }\end{array}$ \\
\hline Mean & 0.08362 & -0.0000035 \\
\hline Standard Deviation & 0.03591 & 0.004063 \\
\hline Monthly $\rho_{1}$ & 0.9389 & 0.02136 \\
\hline$\rho_{2}$ & 0.8785 & -0.00689 \\
\hline$\rho_{3}$ & 0.8300 & -0.01658 \\
\hline$\rho_{4}$ & 0.8014 & 0.00242 \\
\hline p5 & 0.7783 & 0.00858 \\
\hline$\rho_{6}$ & 0.7715 & 0.01573 \\
\hline$\rho_{7}$ & 0.7361 & 0.00056 \\
\hline $\begin{array}{c}\text { Augmented Daily } \\
\text { Dickey-Fuller }\end{array}$ & -2.60 & \\
\hline $\begin{array}{c}\mathbf{H}_{0}: \\
\text { Nonstationary } \\
\end{array}$ & $\begin{array}{c}\text { Reject at } 90 \% \\
\text { (critical value }=-2.57 \text { ) }\end{array}$ & \\
\hline
\end{tabular}

Notes: (i) The augmented Dickey-Fuller test statistics is computed as $\hat{\tau}_{\mu}=\hat{\phi} /$ ase $(\hat{\phi})$ in the model: $\Delta r_{t}=\mu+\phi r_{t-1}+\sum_{j=1}^{p} \phi_{j} \Delta r_{t-1}+u_{t}$, with $p=30$ lags. See e.g., Harvey (1993), section 5.4 .

(ii) The justification for using the Dickey-Fuller table when the residuals are heteroscedastic and possibly serially dependent is provided by Said and Dickey (1984) and Phillips (1987). 


\section{Table 3: Parameter Estimates for the Spot Rate Process}

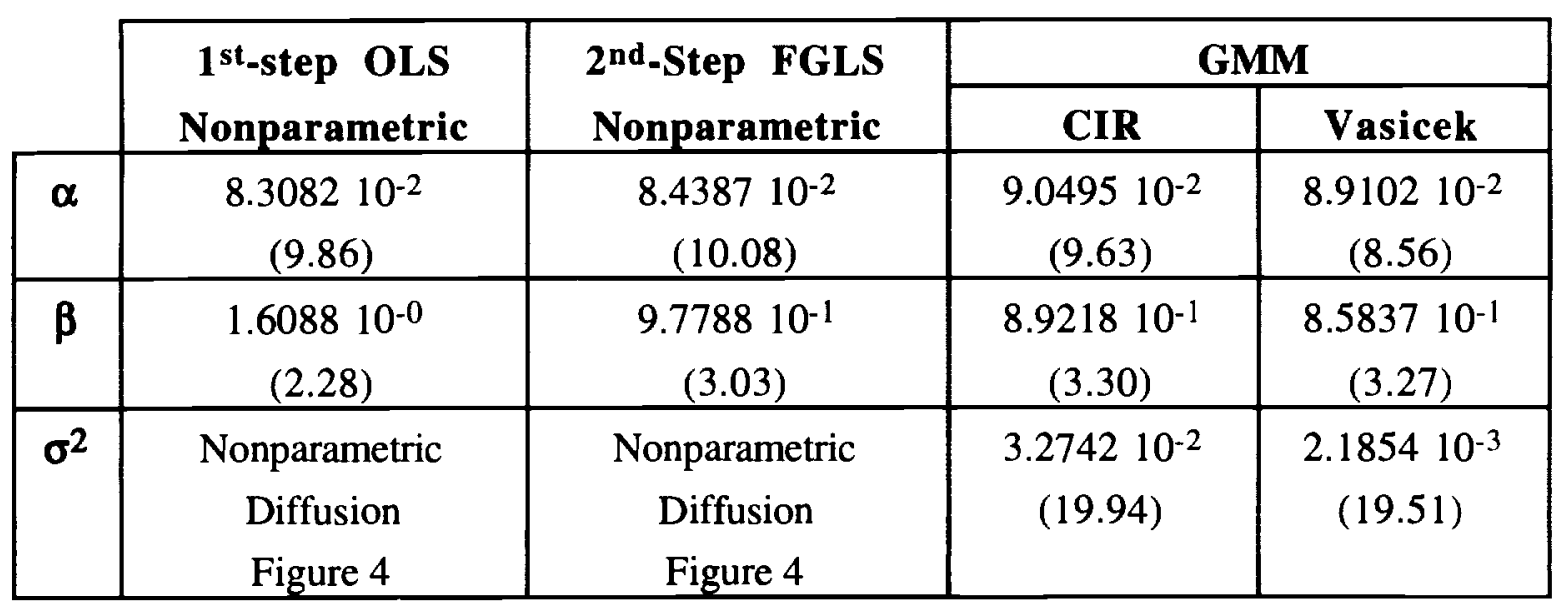

Notes: (i) The estimates reported in the table are for daily sampling of the annualized one-day rate, with time measured in years.

(ii) Heteroskedasticity-robust t-statistics are in parentheses. The t-statistics for OLS use the White's estimator of the asymptotic variance, since in the first step the nature of the heteroskedasticity is unknown.

(iii) The 2-step FGLS estimates were described in Section 3. FGLS uses the first-step nonparametric diffusion function to form the weights and compute the t-statistics.

\section{Table 4: Market Price of Interest Rate Risk}

\begin{tabular}{|c|c|c|c|}
\cline { 2 - 4 } \multicolumn{1}{c|}{} & Nonparametric & CIR & Vasicek \\
\hline$\lambda$ & $\begin{array}{c}-2.445110^{-1} \\
(4.52)\end{array}$ & $\begin{array}{c}-7.890510^{-2} \\
(3.94)\end{array}$ & $\begin{array}{c}-1.698810^{-1} \\
(6.79)\end{array}$ \\
\hline
\end{tabular}

Notes: $\quad$ (i) The market price of risk was estimated by minimizing the squared deviations between the respective model's bond yields and those in Figure 6.

(ii) The bootstrap t-statistic of the estimate is in parentheses. 
Table 5: Nonparametric Underlying Bond Prices

\begin{tabular}{|c|c|c|c|c|c|c|c|}
\hline $\begin{array}{c}\text { Annualized } \\
\text { Spot Rate } \\
\text { Maturity } \\
\text { (Years) } \downarrow\end{array}$ & $\mathbf{0 . 0 2}$ & $\mathbf{0 . 0 4}$ & $\mathbf{0 . 0 6}$ & $\mathbf{0 . 0 8}$ & $\mathbf{0 . 1 0}$ & $\mathbf{0 . 1 2}$ & $\mathbf{0 . 1 4}$ \\
\hline $\mathbf{0 . 5}$ & 98.2408 & 97.4936 & 96.7065 & 95.9064 & 95.1389 & 94.3724 & 93.6150 \\
& $(0.1078)$ & $(0.0940)$ & $(0.0875)$ & $(0.0709)$ & $(0.0861)$ & $(0.0893)$ & $(0.0960)$ \\
& 98.3089 & 97.5054 & 96.7084 & 95.9179 & 95.1339 & 94.3563 & 93.5851 \\
& 98.2859 & 97.4900 & 96.7005 & 95.9175 & 95.1408 & 94.3703 & 93.6061 \\
\hline \multirow{2}{*}{$\mathbf{1}$} & 95.5091 & 94.2250 & 92.9944 & 91.7708 & 90.5475 & 89.3639 & 88.2045 \\
& $(0.1146)$ & $(0.0923)$ & $(0.0839)$ & $(0.0655)$ & $(0.0740)$ & $(0.0832)$ & $(0.0992)$ \\
& 95.6082 & 94.3132 & 93.0357 & 91.7755 & 90.5323 & 89.3060 & 88.0964 \\
& 95.5461 & 94.2721 & 93.0150 & 91.7747 & 90.5509 & 89.3435 & 88.1521 \\
\hline \multirow{2}{*}{$\mathbf{5}$} & 66.8409 & 65.4272 & 64.0544 & 62.7142 & 61.4002 & 60.1130 & 58.8665 \\
& $(0.1125)$ & $(0.1088)$ & $(0.0984)$ & $(0.0664)$ & $(0.0753)$ & $(0.0888)$ & $(0.0991)$ \\
& 67.3719 & 65.7962 & 64.2574 & 62.7546 & 61.2869 & 59.8535 & 58.4537 \\
& 67.2425 & 65.7148 & 64.2218 & 62.7627 & 61.3368 & 59.9433 & 58.5814 \\
\hline & 41.3704 & 40.4903 & 39.5658 & 38.6818 & 37.8343 & 37.0836 & 36.3885 \\
& $(0.0920)$ & $(0.0806)$ & $(0.0724)$ & $(0.0678)$ & $(0.0622)$ & $(0.0706)$ & $(0.0945)$ \\
& 41.5477 & 40.5622 & 39.6000 & 38.6606 & 37.7436 & 36.8483 & 35.9742 \\
& 41.4768 & 40.5218 & 39.5887 & 38.6771 & 37.7865 & 36.9164 & 36.0664 \\
\hline \multirow{2}{*}{$\mathbf{3 0}$} & 5.9825 & 5.8550 & 5.7211 & 5.5802 & 5.4617 & 5.3256 & 5.2232 \\
& $(0.0873)$ & $(0.0752)$ & $(0.0738)$ & $(0.0705)$ & $(0.0814)$ & $(0.0878)$ & $(0.0800)$ \\
& 5.9788 & 5.8369 & 5.6985 & 5.5633 & 5.4313 & 5.3024 & 5.1766 \\
& 5.9759 & 5.8383 & 5.7038 & 5.5724 & 5.4441 & 5.3187 & 5.1962 \\
\hline
\end{tabular}

Notes: $\quad$ (i) All prices correspond to a face value of the bond equal to $\$ 100$. The four elements of each cell are from top to bottom: the nonparametric price, its bootstrap standard error (in parentheses), the CIR price and the Vasicek price.

(ii) The nonparametric prices were computed using the 2-step FGLS estimates of $\alpha$ and $\beta$ (second column of Table 3 ), the nonparametric diffusion estimator (Figure 4) and the market price of risk from Table 4. The CIR and Vasicek prices were computed using the GMM estimates of $\alpha, \beta$ and $\sigma^{2}$ reported in Table 3 (third and fourth column respectively), and the market prices of interest rate risk reported in Table 4.

(iii) The bootstrap standard errors are produced by $\mathrm{nb}=100$ replications with blocks containing $\mathrm{nk}=200$ consecutive spot observations. 
Table 6: Nonparametric Call Option Prices on a 5-Year Bond

\begin{tabular}{|c|c|c|c|c|c|c|}
\hline \multicolumn{2}{|c|}{ Exercise Price $\rightarrow$} & \multirow[t]{2}{*}{0.96} & \multirow[t]{2}{*}{0.98} & \multirow[t]{2}{*}{1.00} & \multirow[t]{2}{*}{1.02} & \multirow[t]{2}{*}{1.04} \\
\hline $\begin{array}{c}\text { Annualized } \\
\text { Spot Rate } \\
\text { (r) } \downarrow \\
\end{array}$ & $\begin{array}{c}\text { Option } \\
\text { Expiration } \\
\text { (t: years) } \downarrow\end{array}$ & & & & & \\
\hline 0.02 & 0.25 & $\begin{array}{c}3.1451 \\
(0.0092) \\
3.1432 \\
3.1513 \\
\end{array}$ & $\begin{array}{c}1.8624 \\
(0.0097) \\
1.8422 \\
1.9061 \\
\end{array}$ & $\begin{array}{c}0.7926 \\
(0.0035) \\
0.7179 \\
0.9049 \\
\end{array}$ & $\begin{array}{c}0.1696 \\
(0.0030) \\
0.0966 \\
0.3071 \\
\end{array}$ & $\begin{array}{c}0.0054 \\
(0.0026) \\
0.0002 \\
0.0694 \\
\end{array}$ \\
\hline 0.02 & 0.5 & $\begin{array}{c}3.8179 \\
(0.0094) \\
3.8071 \\
3.8196 \\
\end{array}$ & $\begin{array}{c}2.5660 \\
(0.0110) \\
2.5379 \\
2.5855 \\
\end{array}$ & $\begin{array}{c}1.4512 \\
(0.0094) \\
1.3910 \\
1.5232 \\
\end{array}$ & $\begin{array}{c}0.6141 \\
(0.0089) \\
0.5231 \\
0.7478 \\
\end{array}$ & $\begin{array}{c}0.1543 \\
(0.0072) \\
0.0865 \\
0.2950 \\
\end{array}$ \\
\hline 0.02 & 1 & $\begin{array}{c}5.5679 \\
(0.0109) \\
5.5574 \\
5.5718 \\
\end{array}$ & $\begin{array}{c}4.3117 \\
(0.0134) \\
4.3061 \\
4.3133 \\
\end{array}$ & $\begin{array}{c}3.1139 \\
(0.0155) \\
3.1056 \\
3.1159 \\
\end{array}$ & $\begin{array}{c}2.0454 \\
(0.0128) \\
2.0077 \\
2.0514 \\
\end{array}$ & $\begin{array}{c}1.1620 \\
(0.0092) \\
1.0911 \\
1.2014 \\
\end{array}$ \\
\hline 0.14 & 0.25 & $\begin{array}{c}4.2449 \\
(0.0125) \\
4.2400 \\
4.2211 \\
\end{array}$ & $\begin{array}{c}3.1665 \\
(0.0156) \\
3.1636 \\
\\
3.0941 \\
\end{array}$ & $\begin{array}{c}2.1716 \\
(0.0127) \\
2.1670 \\
2.0059 \\
\end{array}$ & $\begin{array}{c}1.3281 \\
(0.0148) \\
1.3163 \\
1.0653 \\
\end{array}$ & $\begin{array}{c}0.6962 \\
(0.0124) \\
0.6779 \\
0.4243 \\
\end{array}$ \\
\hline 0.14 & 0.5 & $\begin{array}{c}5.9629 \\
(0.0175) \\
5.9595 \\
5.9392 \\
\end{array}$ & $\begin{array}{c}4.9094 \\
(0.0176) \\
4.8964 \\
4.8437 \\
\end{array}$ & $\begin{array}{c}3.8868 \\
(0.0152) \\
3.8679 \\
\\
3.7558 \\
\end{array}$ & $\begin{array}{c}2.9515 \\
(0.0156) \\
2.9010 \\
2.7009 \\
\end{array}$ & $\begin{array}{c}2.0940 \\
(0.0139) \\
2.0322 \\
1.7418 \\
\end{array}$ \\
\hline 0.14 & 1 & $\begin{array}{c}9.0293 \\
(0.0194) \\
9.0241 \\
9.0063 \\
\end{array}$ & $\begin{array}{c}8.0119 \\
(0.0187) \\
8.0013 \\
7.9735 \\
\end{array}$ & $\begin{array}{c}6.9534 \\
(0.0176) \\
6.9857 \\
6.9408 \\
\end{array}$ & $\begin{array}{c}5.9949 \\
(0.0175) \\
5.9828 \\
5.9089 \\
\end{array}$ & $\begin{array}{r}5.0125 \\
(0.0163) \\
5.0019 \\
4.8809 \\
\end{array}$ \\
\hline
\end{tabular}

Notes: (i) All call option prices correspond to a face value of the discount bond equal to $\$ 100$. The exercise price is expressed as a proportion of the corresponding bond price for each model.

(ii) The four elements of each cell are from top to bottom: the nonparametric price, its bootstrap standard error in parentheses, the closed-form CIR price and the closedform Vasicek price. The standard errors of the nonparametric prices were computed under the same conditions as Table 5. 
Figure 1

Spot Interest Rate

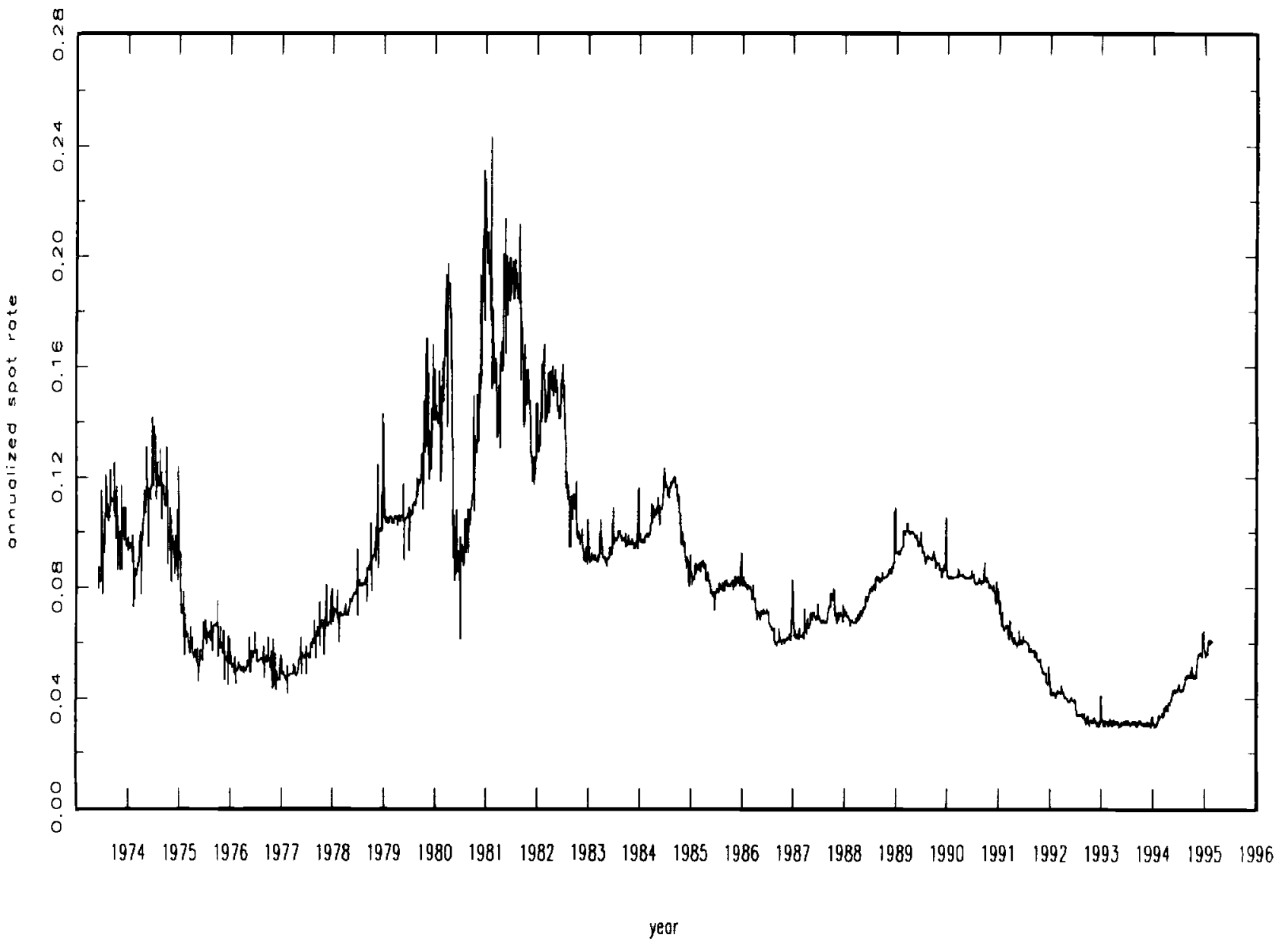


Figure 2

Nonparometric Kernel Density. CIR and Vasicek Densities

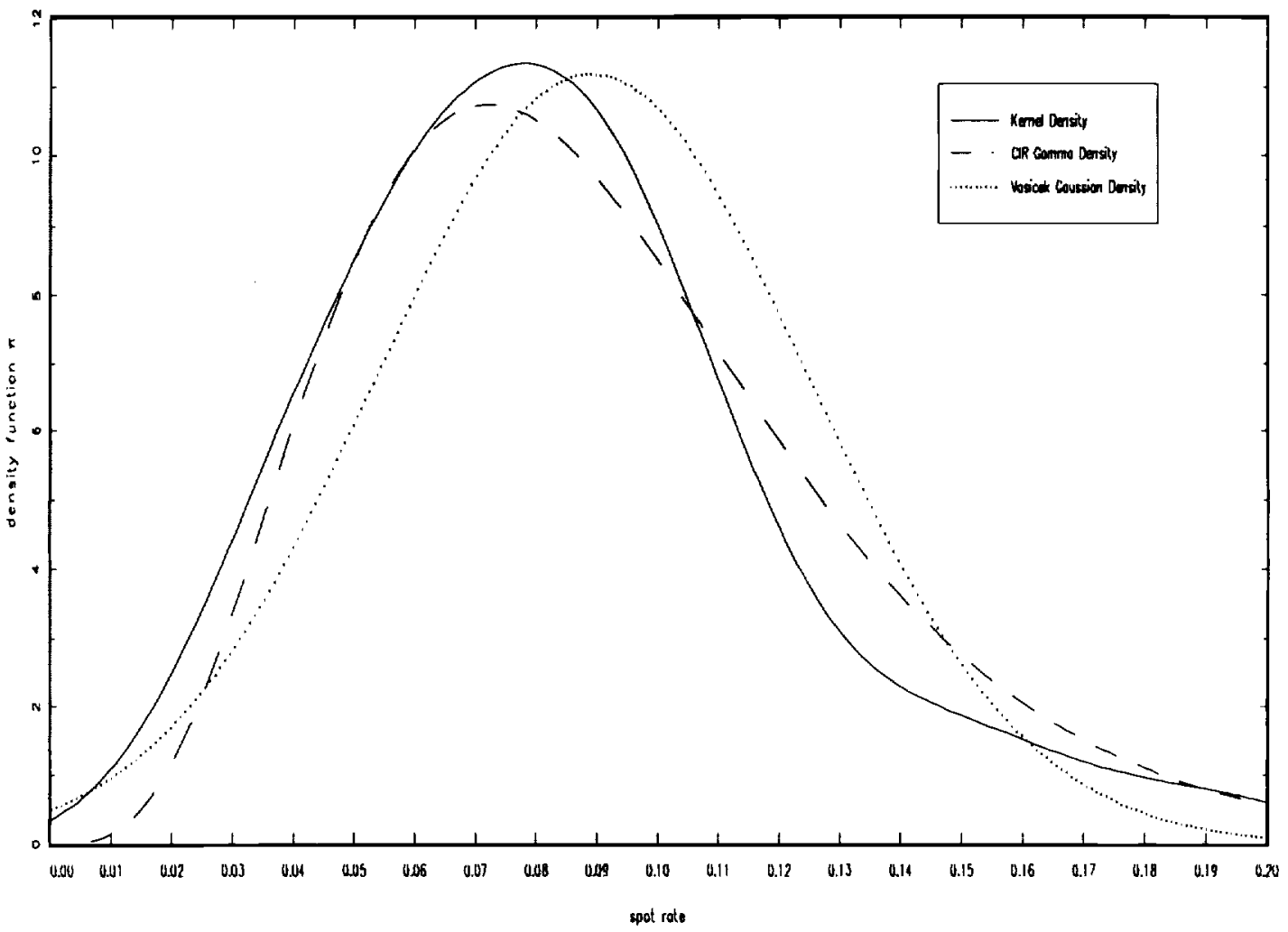

Figure 3

Nonporometric Kernel Density with 997. Confidence Band

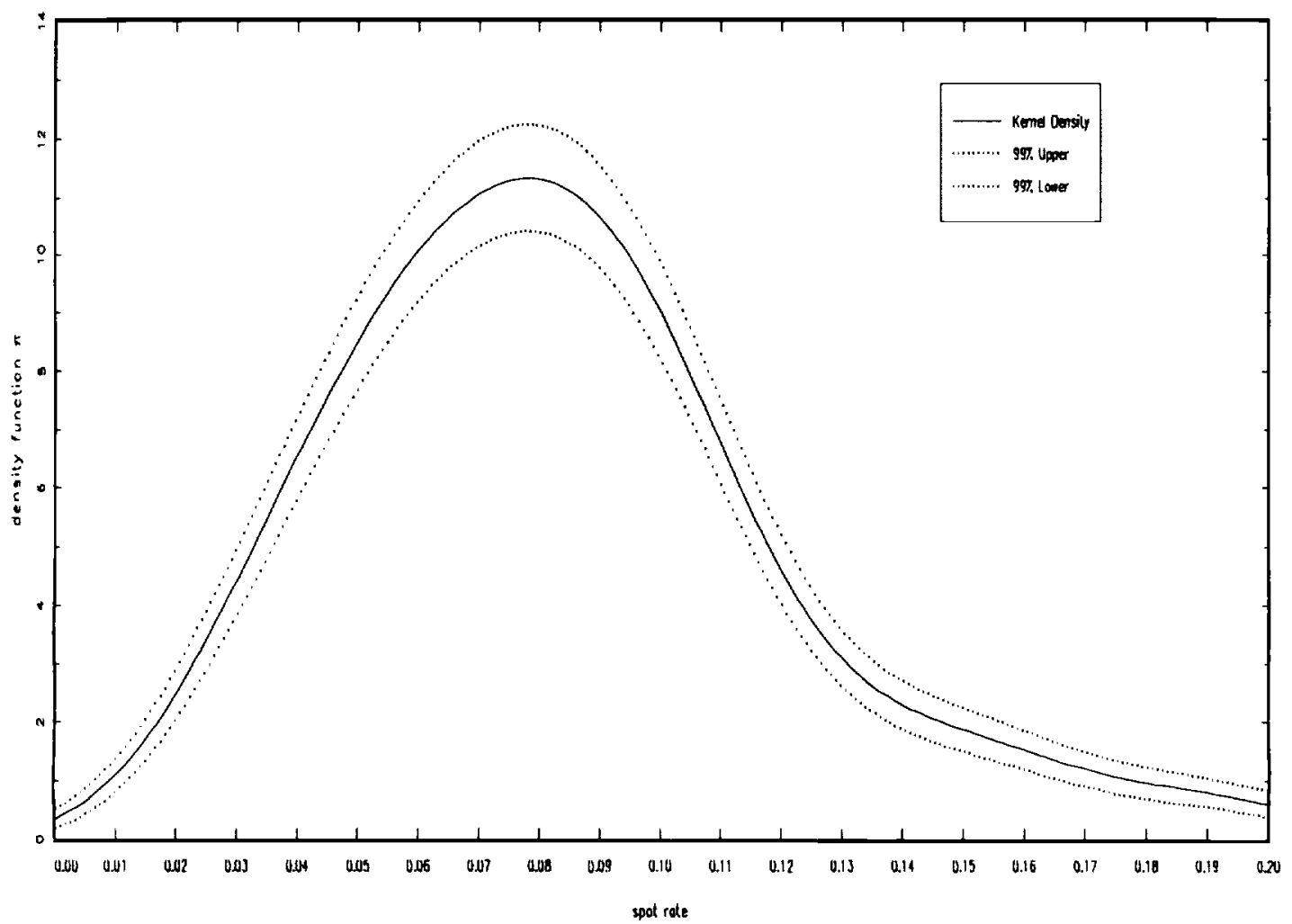


Figure 4

Nonporametric Diffusion Estimotor Compared to CIR and Vosicek

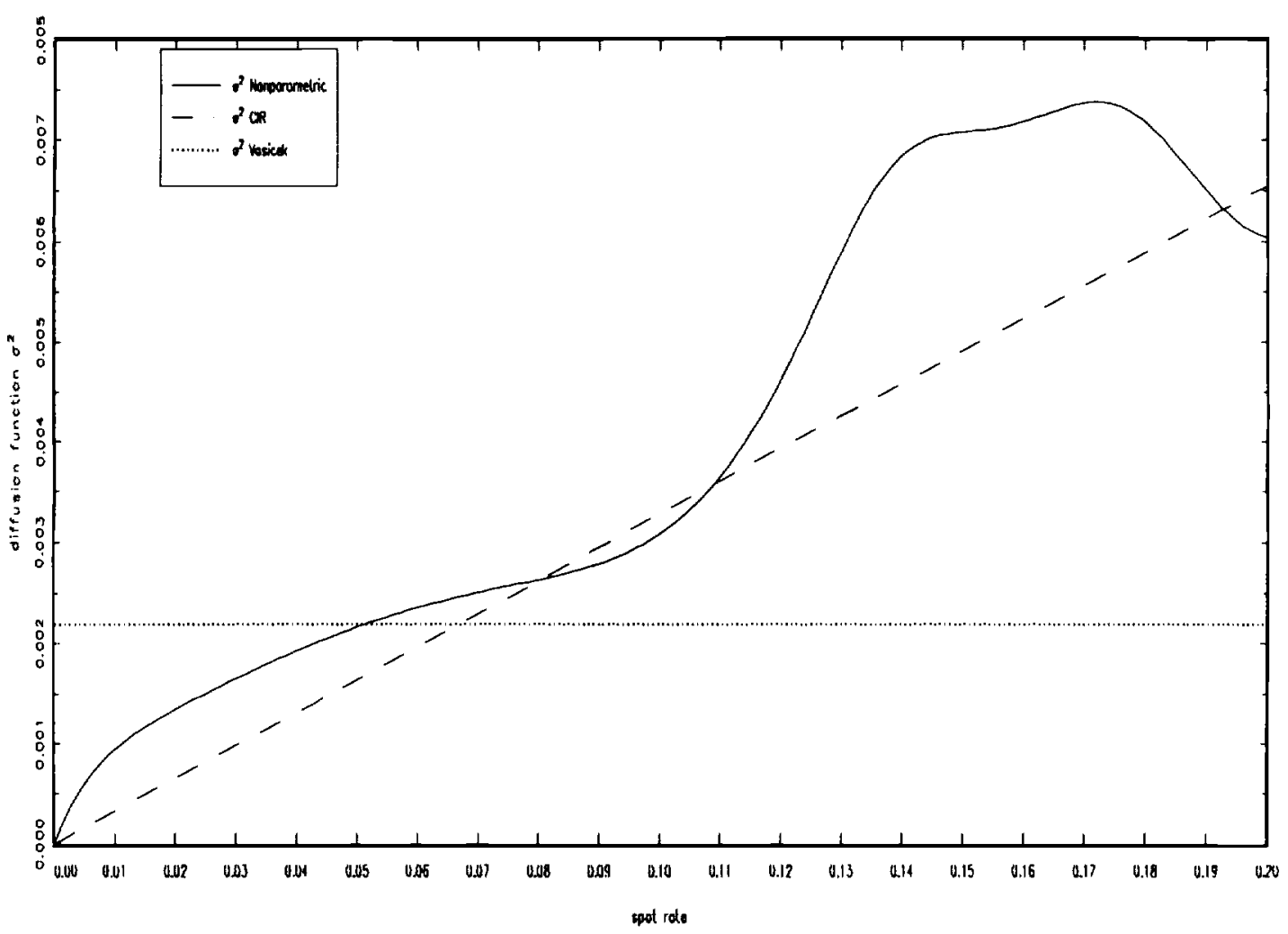

Figure 5

Nonporametric Diffusion Estimotor with 957. Confidence Bond

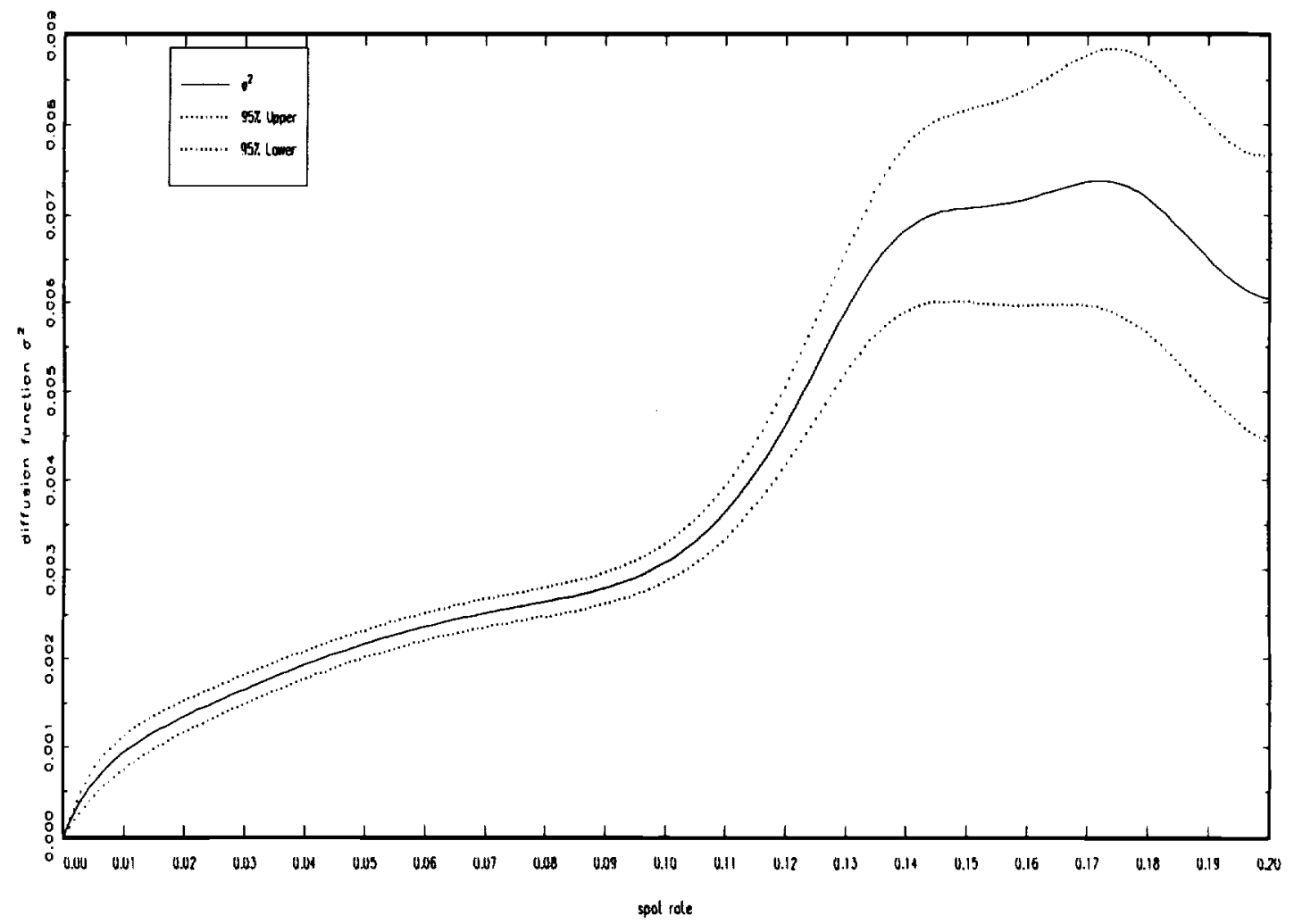


Figure 6

Yield Curve

(spot rote $\mathrm{r}=0.07$ )

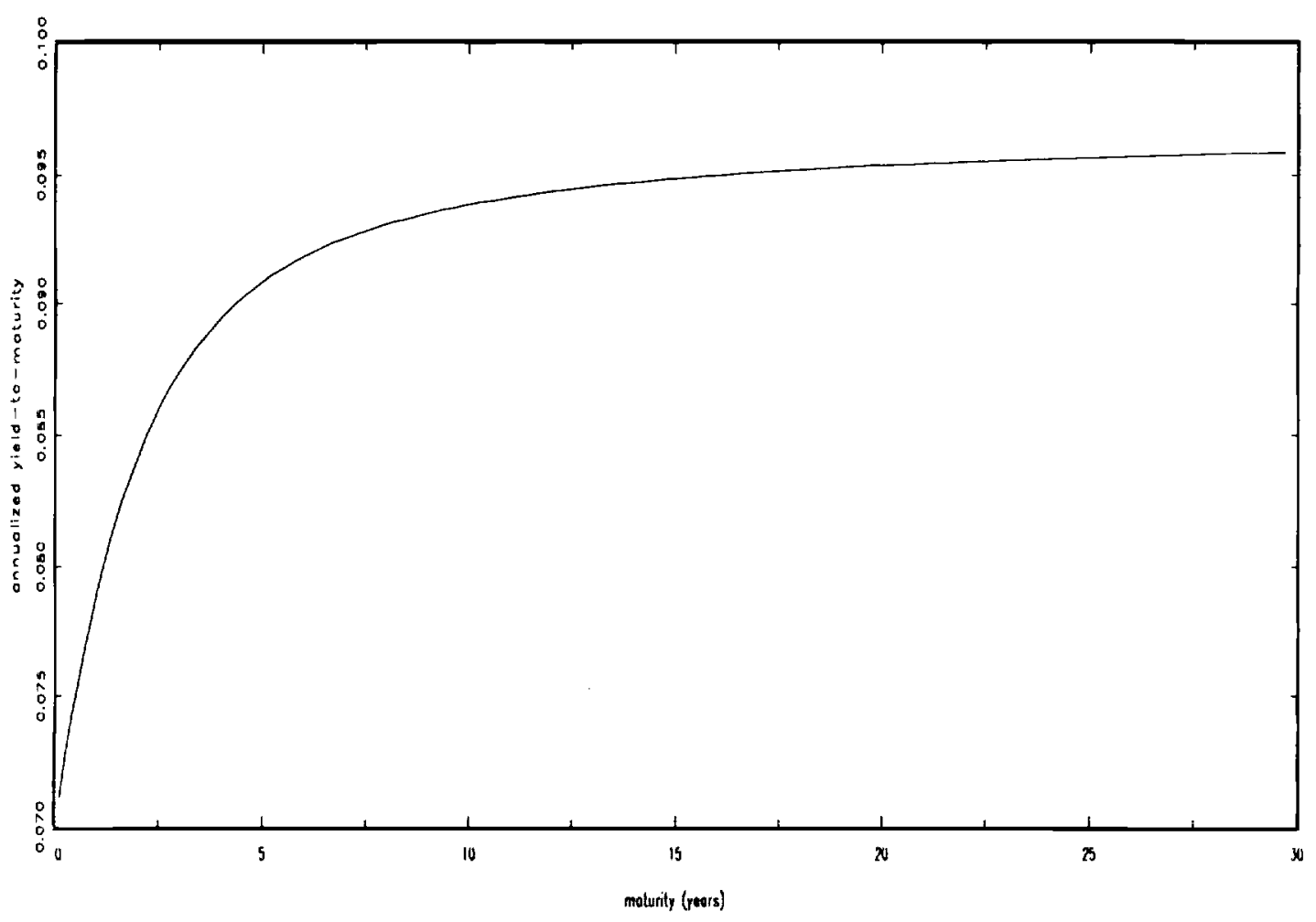

Figure 7

5-yeor Bond Price Volatilities

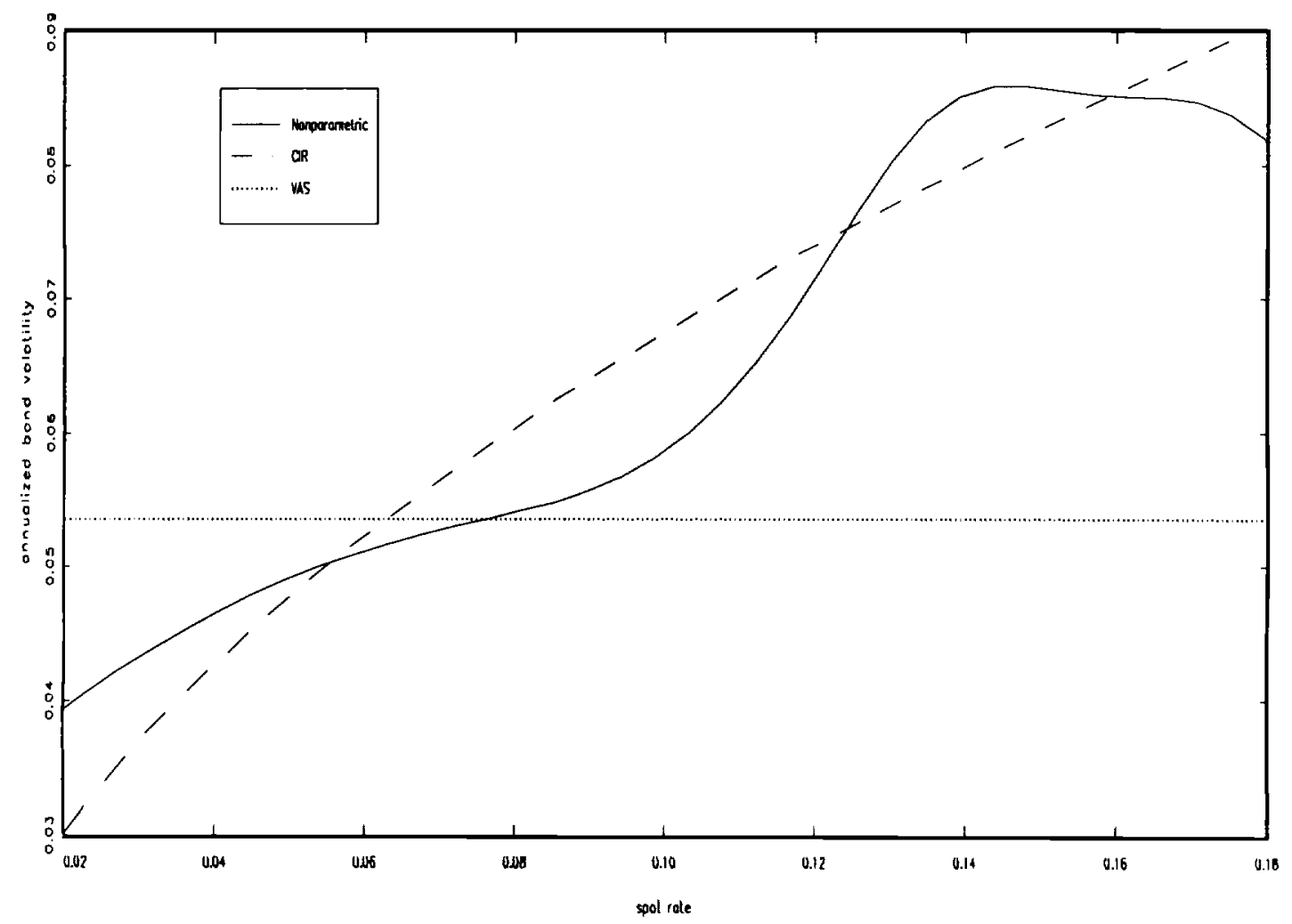

